\title{
Heisenberg Evolution WKB and Symplectic Area Phases
}

\author{
T. A. Osborn \\ Department of Physics and Astronomy \\ University of Manitoba \\ Winnipeg, MB, Canada, R3T 2N2 \\ M. F. Kondratieva \\ Department of Mathematics and Statistics \\ University of Minnesota \\ Duluth, MN, USA 55812
}

\begin{abstract}
The Schrödinger and Heisenberg evolution operators are represented in phase space $T^{*} \mathbb{R}^{n}$ by their Weyl symbols. Their semiclassical approximations are constructed in the short and long time regimes. For both evolution problems, the WKB representation is purely geometrical: the amplitudes are functions of a Poisson bracket and the phase is the symplectic area of a region in $T^{*} \mathbb{R}^{n}$ bounded by trajectories and chords. A unified approach to the Schrödinger and Heisenberg semiclassical evolutions is developed by introducing an extended phase space $\chi_{2} \equiv T^{*}\left(T^{*} \mathbb{R}^{n}\right)$. In this setting Maslov's pseudodifferential operator version of WKB analysis applies and represents these two problems via a common higher dimensional Schrödinger evolution, but with different extended Hamiltonians. The evolution of a Lagrangian manifold in $\chi_{2}$, defined by initial data, controls the phase, amplitude and caustic behavior. The symplectic area phases arise as a solution of a boundary condition problem in $\chi_{2}$. Various applications and examples are considered. Physically important observables generally have symbols that are free of rapidly oscillating phases. The semiclassical Heisenberg evolution in this context has been traditionally realized as an $\hbar$ power series expansion whose leading term is classical transport. The extended Heisenberg Hamiltonian has a reflection symmetry that ensures this behavior. If the WKB initial phase is zero, it remains so for all time, and semiclassical dynamics reduces to classical flow plus finite order $\hbar$ corrections.
\end{abstract}




\section{Introduction}

The version of quantum mechanics most suitable for semiclassical dynamics is Moyal's [1] representation. This classical like statement of quantum mechanics is achieved by the Wigner-Weyl [2, 3] correspondence, which uniquely associates Hilbert space operators with Weyl symbols (functions) on phase space. This correspondence transforms the non-commutative product of operators into a non-commutative $*$ product of symbols. Expectation values are realized as integrals over phase space.

For systems whose coordinate manifold is flat, the Wigner-Weyl isomorphism is most compactly described [4, 6, 6] in terms of the quantizer. Let $T(x)=\exp [i(p \cdot \hat{q}-q \cdot \hat{p}) / \hbar]$ be the Heisenberg translation operator. Here $x=(q, p)$ are the position, momentum coordinates of the linear phase space $\chi_{1} \equiv T^{*} \mathbb{R}_{q}^{n}$, and $\hat{x}=(\hat{q}, \hat{p})$ are the corresponding quantum counterparts. The parity operator on Hilbert space is $P \psi(q)=\psi(-q), \psi \in L^{2}\left(\mathbb{R}_{q}^{n}\right)$. The product of these two unitary operators defines the quantizer, $\Delta(x) \equiv 2^{n} T(2 x) P$. In terms of $\Delta(x)$, the one-to-one Weyl symbol-operator pairing is

$$
\widehat{A}=h^{-n} \int_{\chi_{1}} d x A(x) \Delta(x), \quad A(x)=[\widehat{A}]_{\mathrm{w}}(x)=\operatorname{Tr} \widehat{A} \Delta(x),
$$

where $h=(2 \pi \hbar)$. The first statement above is Weyl quantization; while the second is de-quantization. Whenever the operator is a density matrix $\hat{\rho}$, the quantity $h^{-n} \rho(x)$ is the Wigner distribution. The above map, from $\widehat{A}$ to $A(x)$, gives the same result as the Wigner transform (A.3) of the kernel $\left\langle q|\widehat{A}| q^{\prime}\right\rangle$.

The star product is defined by the requirement that $[\widehat{A} \widehat{B}]_{\mathrm{w}}=A * B$ cf. Appendix A. For small $\hbar$, the $*$ multiplication is approximately the commutative product of symbols,

$$
A * B(x)=A(x) B(x)+i \hbar / 2\{A, B\}(x)+O\left(\hbar^{2}\right) .
$$

The Poisson bracket term measures, to leading $\hbar$ order, the non-commutative character of the star product.

The two basic statements of quantum dynamics are the Schrödinger and Heisenberg pictures. On the space, $L^{2}\left(\mathbb{R}_{q}^{n}\right)$, each self-adjoint Hamiltonian $\widehat{H}$ generates a unitary Schrödinger evolution, $\widehat{U}(t)=\exp [-i t \widehat{H} / \hbar]$. An initial density matrix $\hat{\rho}_{0}$ has Heisenberg evolution, $\hat{\rho}(t)=\widehat{U}(t) \hat{\rho}_{0} \widehat{U}(t)^{\dagger}$. Let $H, U(t)$ and $\rho(t)$ be the Weyl symbols of $\widehat{H}, \widehat{U}(t)$ and $\hat{\rho}(t)$, respectively. In this setting the equations of motion are

$$
\begin{aligned}
i \hbar \partial U(t, x) / \partial t & =H(x) * U(t, x), \\
\partial \rho(t, x) / \partial t & =\{H, \rho(t)\}_{M}(x),
\end{aligned}
$$

with initial conditions $U(0, x)=1$, and $\rho(0, x)=\rho_{0}(x)$. In (1.2), the bracket operation is defined by

$$
\{A, B\}_{M} \equiv \frac{1}{i \hbar}[\widehat{A}, \widehat{B}]_{\mathrm{w}}=\frac{1}{i \hbar}(A * B-B * A) .
$$

This is the Moyal bracket. Like the quantum commutator, it is bilinear, skew and obeys the Jacobi identity. 
It is evident that the Weyl-Heisenberg symbol evolution problem (1.2) is formally as close as it can be to classical dynamics. Since $\{A, B\}_{M}=\{A, B\}+O\left(\hbar^{2}\right)$, Bohr's correspondence principle is realized in a transparent form. The time dependent expectation value of an observable $\widehat{A}$ is conveniently given by the phase space integral

$$
\langle\widehat{A}\rangle_{t} \equiv \operatorname{Tr} \widehat{A} \hat{\rho}(t)=h^{-n} \int_{\chi_{1}} d x A(x) \rho(t, x) .
$$

The main goal of this paper is to obtain both short and long time WKB approximate solutions for the Weyl symbol evolution problems (1.1) and (1.2). In Theorems 1 and 2 , we prove that the phases entering these semi-classical representations are invariant geometrical quantities - namely the symplectic areas defined by certain closed loops in phase space. Furthermore, the amplitude functions are given by determinants of a Poisson bracket.

We employ a common method to solve both problems (1.1) and (1.2). Standard Weyl symbol calculus identities show that $H *$ and $\{H, \cdot\}_{M}$ are pseudodifferential operators, in other words they are functions of $\nabla_{x}$ and multiplication by $x$. If one introduces an extended phase space $\chi_{2} \equiv T^{*}\left(T^{*} \mathbb{R}_{q}^{n}\right)$, then Maslov's [7] WKB analysis applies. In particular, the semiclassical approximations for the evolutions $U(t, x)$ and $\rho(t, x)$ are characterized by a Lagrangian manifold, and the time evolution of this manifold is determined by an extended Hamiltonian in $\chi_{2}$. In order to distinguish these two phase spaces we call $\chi_{1}$ the primary phase space (PPS), and $\chi_{2}$ the secondary phase space (SPS). Projecting the $\chi_{2}$ analysis back onto the primary phase space provides explicit WKB representations of $U(t, x)$ and $\rho(t, x)$.

The results in the literature nearest to this paper are found in three seminal works: by Karasev and Nazaikinskii [8], Berry [9], and Marinov [10]. In the first of these, an analytical approach to a generalized semiclassical expansion based on a SPS with its associated left-right representation of $*$-product and Hamilton-Jacobi equation was developed. In the second pair of works, the geometrical (symplectic phase) interpretation of the Wigner eigenfunction and of the dynamical WKB approximation was achieved. Specifically, in the small time sector, where the time evolving $\chi_{2}$ Lagrangian manifold remains single sheeted, Marinov found the WKB amplitude and phase for $U(t, x)$. In addition to providing an entirely different proof, our treatment extends these known results in several ways. First, we determine the long time version of the WKB approximation where the $\chi_{2}$ Lagrangian manifold may be multi-sheeted with several points corresponding to a given $x \in \chi_{1}$. Second, by incorporating Maslov's results one maintains control of the error estimates. Third, we solve a generalized version of (1.1) with rapidly oscillating initial data. This latter generalization arises for the evolution $\widehat{U}\left(t+t_{0}\right), t_{0}>0$. At $t=0$ the initial data generically will have the rapidly oscillating form, $N_{0}(x) e^{i \Phi_{0}(x) / \hbar}$.

In spite of the importance of problem (11.2), it has received insufficient study. The version of the Heisenberg problem that has been investigated in detail [11, 12, 13, 14] occurs for the case where the observable $\widehat{A}$ is semiclassically admissible, namely its Weyl symbol is $\hbar$ dependent and allows a finite order small $\hbar$ asymptotic expansion $c f$. (3.6). In [15] a connected graph method was constructed to obtain the coefficients of the $\hbar$ asymptotic expansion of $\left[\widehat{U}(t)^{\dagger} \widehat{A} \widehat{U}(t)\right]_{\mathrm{w}}(x)$. Based on this latter formalism, numerical calculations have shown [16] that the $\hbar^{2}$-order semiclassical expansion accurately describes 
noble gas atom-atom scattering. However, semiclassically admissible symbols are not suitable for representing [17] density matrices. The requirement that one has a pure state, $\rho_{0} * \rho_{0}=\rho_{0}$, means that $\rho_{0}(x)$ is an $\hbar \rightarrow 0$ rapidly oscillating function $c f$. (2.12).

The paper has the following organization. Section 2 presents the extended Schrödinger equation in the SPS setting and shows how the problems (1.1) and (1.2) are particular examples of this extended evolution. Also, the geometric, commutative and coordinate relationships between the PPS and SPS are reviewed. The trajectories entering the WKB expansion are determined by the solutions of a boundary condition problem. In Section 3, a detailed analysis of the $\mathrm{BC}$ problem and the manner in which it constructs phase space loops is carried out. The semiclassical expansions for $U(t, x)$ and $\rho(t, x)$ are consolidated in Theorems 1 and 2. Finally, in Section 4 the group generated phase addition rules are obtained; the mutual compatibility of the Schrödinger and Heisenberg semiclassical evolution is established; the exact solutions for quadratic Hamiltonian systems are compared; and, the $\hbar \rightarrow 0$ asymptotics of $\left[\widehat{U}(t) \widehat{A} \widehat{U}(t)^{\dagger}\right]_{\mathrm{w}}(x)$ for semiclassically admissible operators $\widehat{A}$ is realized as a special case of Theorem 2 . There are three appendices containing specialized aspects of Weyl symbol calculus, pseudodifferential operators, Jacobi fields, and a Poincaré-Cartan identity.

\section{Secondary Phase Space Dynamics}

In the first part of this section we show how to interpret the $H *$ structure in the equations of motion as the action of a pseudodifferential operator ( $\Psi D O)$. The normal ordered symbols of these operators are then functions on the secondary phase space. For the Schrödinger and Heisenberg problem we show how the classical flow in $\chi_{2}$ is composed in terms of the standard Hamiltonian mechanics on $\chi_{1}$. The necessity of representing the initial value form of the density matrix as a rapidly oscillating $\hbar$ function is confirmed by constructing the semiclassical Weyl symbol for a pure state.

Introduce the SPS canonical operators $X, Y$ acting on functions $f(x): X f(x)=x f(x)$ and $Y f(x)=-i \hbar \nabla_{x} f(x)$. Let $\mathcal{H}(\stackrel{2}{X}, \stackrel{1}{Y})$ be a normal ordered, symmetric $\Psi \mathrm{DO}$ defined by the symbol $\mathcal{H}(x, y)$. On the coordinate domain $\mathbb{R}_{x}^{2 n}$, the extended Schrödinger evolution problem is

$$
i \hbar \partial \Psi(t, x) / \partial t=\mathcal{H}(\stackrel{2}{X}, \stackrel{1}{Y}) \Psi(t, x) .
$$

The two problems (1.1) and (1.2) are particular cases of (2.1). The appropriate realization of $\mathcal{H}$ in each case is determined by employing the left-right $*$ product representation [8, 18] (see Appendix A). Setting $\mathcal{H}(x, y)=\mathcal{H}_{1} \equiv H\left(x-\frac{1}{2} J y\right)$ selects the Schrödinger problem (1.1), whereas the choice $\mathcal{H}_{2} \equiv H\left(x-\frac{1}{2} J y\right)-H\left(x+\frac{1}{2} J y\right)$ gives the Heisenberg problem (1.2). The solution $\Psi(t, x)$ is either $U(t, x)$ or $\rho(t, x)$ depending on the choice of $\mathcal{H}$. In $\mathrm{SPS}$, the scalar function $\mathcal{H}$ is a Hamiltonian which is the generator of $\chi_{2}$ classical flow.

Consistency of this extended Schrödinger problem requires that the normal ordered symbol $H\left(x \pm \frac{1}{2} J y\right)$ define a symmetric operator on $L^{2}\left(\mathbb{R}_{x}^{2 n}\right)$. That is does so is a consequence of the argument structure $\left(x \pm \frac{1}{2} J y\right.$ ) which implies that $c f$. (A.16) the normal ordered and Weyl ordered symbols are the same function. Since the Weyl ordered symbol is real, one has that the $\Psi \mathrm{DO}$ Hamiltonians in (2.1) are formally self-adjoint. 
Consider the coordinate systems of $\chi_{1}$ and $\chi_{2}$ and their interconnections. Let $y$ be the momentum variable in SPS so that a general point $m \in \chi_{2}$ has Cartesian coordinates $z(m)=(x, y)$. The symplectic structure of $\chi_{1}$ and $\chi_{2}$ is determined by their respective canonical 2-forms

$$
\begin{gathered}
\omega^{(1)} \equiv d p \wedge d q=\frac{1}{2} J_{\alpha \beta} d x_{\beta} \wedge d x_{\alpha}, \quad J=\left[\begin{array}{cc}
0 & I_{n} \\
-I_{n} & 0
\end{array}\right], \\
\omega^{(2)} \equiv d y \wedge d x=\frac{1}{2} \widetilde{J}_{j k} d z_{k} \wedge d z_{j},
\end{gathered}
$$

where $I_{n}$ is the $n \times n$ identity matrix, and $\widetilde{J}$ is the symplectic matrix having the block form of $J$ with $I_{2 n}$ substituted for $I_{n}$. Given the 2-forms, $\omega^{(1)}$ and $\omega^{(2)}$, the corresponding Poisson brackets are

$$
\{f, g\}_{1}(x)=\nabla f(x) \cdot J \nabla g(x), \quad\{F, G\}_{2}(z)=\nabla F(z) \cdot \widetilde{J} \nabla G(z) .
$$

The coordinate functions, $x=(q, p)$ and $z=(x, y)$, are canonical in $\chi_{1}$ and $\chi_{2}$, namely $\left\{x_{\alpha}, x_{\beta}\right\}_{1}=J_{\alpha \beta}$ and $\left\{z_{j}, z_{k}\right\}_{2}=\widetilde{J}_{j k}$. In order that (2.1) be a standard Schrödinger equation the coordinates $x=(q, p)$ must commute. This is the case since $\left\{x_{\alpha}, x_{\beta}\right\}_{2}=0$.

As one sees from functional forms of $\mathcal{H}$, a second natural coordinate system for $\chi_{2}$ is defined by the $(l, r)$ variables

$$
\begin{aligned}
& l=x-\frac{1}{2} J y, \quad r=x+\frac{1}{2} J y, \quad l, r \in \mathbb{R}^{2 n}, \\
& x=\frac{1}{2}(l+r), \quad y=J(l-r) .
\end{aligned}
$$

The link between the coordinate systems $(x, y)$ and $(l, r)$, and their commutative structures is the following.

Lemma 1. (i) The SPS commutative properties of the projections $l, r: \mathbb{R}_{z}^{4 n} \rightarrow \mathbb{R}_{x}^{2 n}$ are

$$
\left\{l_{\alpha}, l_{\beta}\right\}_{2}=J_{\alpha \beta}, \quad\left\{r_{\alpha}, r_{\beta}\right\}_{2}=-J_{\alpha \beta}, \quad\left\{l_{\alpha}, r_{\beta}\right\}_{2}=0 .
$$

(ii) The $l$ variables are Poisson; the $r$ variables are anti-Poisson, namely

$$
\{f \circ l, g \circ l\}_{2}=\{f, g\}_{1} \circ l, \quad\{f \circ r, g \circ r\}_{2}=-\{f, g\}_{1} \circ r .
$$

(iii) The $\chi_{2}$ Poisson bracket and symplectic form have the $l \times r$ decomposition

$$
\begin{gathered}
\{F, G\}_{2}=\left(\nabla_{l} F\right)_{\alpha} J_{\alpha \beta}\left(\nabla_{l} G\right)_{\beta}-\left(\nabla_{r} F\right)_{\alpha} J_{\alpha \beta}\left(\nabla_{r} G\right)_{\beta}, \\
\widetilde{J}_{j k} d z_{k} \wedge d z_{j}=J_{\alpha \beta} d l_{\beta} \wedge d l_{\alpha}-J_{\alpha \beta} d r_{\beta} \wedge d r_{\alpha} .
\end{gathered}
$$

The space structure for the left-right variables is $\chi_{1} \otimes \chi_{1}^{\prime} \ni(l, r)$. Here $\chi_{1}^{\prime}$ denotes the phase space defined by the 2 -form $-\frac{1}{2} J_{\alpha \beta} d r_{\beta} \wedge d r_{\alpha}$. Often the space $\chi_{1} \otimes \chi_{1}^{\prime}$ is labelled [19] the double phase space. We use the name secondary phase space for cotangent bundle $\chi_{2}=T^{*}\left(T^{*} R_{q}^{n}\right)$ in order to distinguish it from $\chi_{1} \otimes \chi_{1}^{\prime}$. The left-right map, (2.2) is diffeomorphic.

The basic ingredients in any semiclassical approximation are the classical action phase and the amplitude functions determined by classical transport. Because the extended 
Hamiltonians are the simple composite functions $H \circ l$ and $H \circ l-H \circ r$, it is possible to describe the $\chi_{2}$ phase space motion as appropriately weighted sums of $\chi_{1}$ trajectories. Let $g\left(\tau \mid x_{0}\right)=\left(q\left(\tau \mid x_{0}\right) ; p\left(\tau \mid x_{0}\right)\right)$ denote the solution of the PPS Hamiltonian system

$$
\dot{g}\left(\tau \mid x_{0}\right)=J \nabla H\left(g\left(\tau \mid x_{0}\right)\right), \quad g\left(0 \mid x_{0}\right)=x_{0} .
$$

The SPS motion is labelled $G\left(\tau \mid z_{0}\right)=\left(x\left(\tau \mid z_{0}\right) ; y\left(\tau \mid z_{0}\right)\right)$, where

$$
\dot{G}\left(\tau \mid z_{0}\right)=\widetilde{J} \nabla \mathcal{H}\left(G\left(\tau \mid z_{0}\right)\right), \quad G\left(0 \mid z_{0}\right)=z_{0}
$$

The initial data $z_{0}$ for system (2.4) also has the left-right representation $l_{0}=l\left(z_{0}\right), r_{0}=$ $r\left(z_{0}\right)$.

Lemma 2. The solution of the SPS Hamiltonian system, in terms of $\chi_{1}$ flows, takes the form: (i) For the Schrödinger problem with $\mathcal{H}_{1}=H \circ l$

$$
x\left(\tau \mid z_{0}\right)=\frac{1}{2}\left(g\left(\tau \mid l_{0}\right)+r_{0}\right), \quad y\left(\tau \mid z_{0}\right)=J\left(g\left(\tau \mid l_{0}\right)-r_{0}\right) .
$$

(ii) For the Heisenberg problem with $\mathcal{H}_{2}=H \circ l-H \circ r$

$$
x\left(\tau \mid z_{0}\right)=\frac{1}{2}\left(g\left(\tau \mid l_{0}\right)+g\left(\tau \mid r_{0}\right)\right), \quad y\left(\tau \mid z_{0}\right)=J\left(g\left(\tau \mid l_{0}\right)-g\left(\tau \mid r_{0}\right)\right) .
$$

Proof. Consider part (i). Using the Poisson equations of motion, it is convenient to state the SPS flow in the $l, r$ variables. Lemma 1(iii) implies

$$
\dot{l}_{\alpha}(\tau)=\left\{l_{\alpha}, H \circ l\right\}_{2}=J_{\alpha \beta} \nabla_{\beta} H(l(\tau)), \quad \dot{r}_{\alpha}(\tau)=\left\{r_{\alpha}, H \circ l\right\}_{2}=0 .
$$

So $l\left(\tau \mid l_{0}\right)=g\left(\tau \mid l_{0}\right)$ and the $r$-motion is a constant, $r\left(\tau \mid r_{0}\right)=r_{0}$. Using (2.2) recovers (2.5). A similar argument verifies part (ii).

The identities (2.7) show that the left-right motions are decoupled. This decoupling occurs because $\left\{l_{\alpha}(\tau), r_{\beta}(\tau)\right\}_{2}=0$. Further note that the system $\mathcal{H}_{1}=H \circ l$ has $2 n$ constants of motion; $r_{\alpha}(\tau)=$ const for $\alpha=1 \ldots 2 n$. Nevertheless, $\mathcal{H}_{1}$ is not completely integrable since the functions $r_{\alpha}(\tau)$ are not in involution, i.e. $\left\{r_{\alpha}(\tau), r_{\beta}(\tau)\right\}_{2}=-J_{\alpha \beta} \neq 0$.

Lagrangian manifolds in SPS create canonical transformations in PPS. Let us clarify how this is realized in terms the left right projections. Suppose $\Lambda$ is a $\chi_{2}$ manifold which is locally defined by the smooth phase $S: U \subseteq \mathbb{R}_{x}^{2 n} \rightarrow \mathbb{R}$, i.e. $y=\nabla S(x), x \in U$. This identity relates $l$ to $r$ by

$$
r-l=J \nabla S\left(\frac{1}{2}(l+r)\right) .
$$

If $\operatorname{det}\left(I \pm \frac{1}{2} J S^{\prime \prime}\right) \neq 0, x \in U$ then the implicit function theorem defines $l=l(r)$ and $r=r(l)$. These transformations are canonical. This follows from

$$
\frac{d l}{d r}=\frac{\left(I-\frac{1}{2} J S^{\prime \prime}\right)}{\left(I+\frac{1}{2} J S^{\prime \prime}\right)} .
$$

The right side above is the Cayley transform of the symmetric $S^{\prime \prime}$ and so defines a symplectic matrix. A systematic study of generating functions of this type is found in [20] 
An important aspect of WKB analysis is the $\hbar$ singularity structure of the Cauchy initial data. As noted in the Introduction, a pure state density matrix must be a rapidly oscillating function. Let us clarify this situation with an example. Suppose $\psi_{0}(q)=$ $n(q) \exp [i s(q) / \hbar]$ is a unit normalized $L^{2}\left(\mathbb{R}_{q}^{n}\right)$ wave function, which defines a rank one density matrix, $\hat{\rho}_{0}=\left|\psi_{0}\right\rangle\left\langle\psi_{0}\right|$. The functions $n, s$ are real. The Weyl symbol of $\hat{\rho}_{0}$ is given by the Wigner transform (A.3)

$$
\begin{aligned}
\rho_{0}(x ; \hbar) & =\int_{\mathbb{R}^{n}} d v n\left(q+\frac{1}{2} v\right) n\left(q-\frac{1}{2} v\right) \exp \frac{i}{\hbar} \phi(v, x), \\
\phi(v, x) & =s\left(q+\frac{1}{2} v\right)-s\left(q-\frac{1}{2} v\right)-p \cdot v .
\end{aligned}
$$

The $\hbar \rightarrow 0$ asymptotic form of $\rho_{0}$ may be calculated by a stationary phase approximation [7]. Let $\phi^{\prime}(v, x)=\nabla_{v} \phi(v, x)$ and $\phi^{\prime \prime}(v, x)=\nabla_{v} \nabla_{v} \phi(v, x)$. The critical points of $\phi$ satisfy

$$
2 p=\nabla s\left(q-\frac{1}{2} v\right)+\nabla s\left(q+\frac{1}{2} v\right) .
$$

Suppose $\left(v_{0}, x_{0}\right)$ is a solution set for this equation where $\operatorname{det} \phi^{\prime \prime}\left(v_{0}, x_{0}\right) \neq 0$, then the implicit function theorem defines a function $v=v(q, p)=v(x)$ obeying (2.11). Since $\phi^{\prime}(v, x)$ is even in $v$, the roots occur in pairs. If $v=v(x)$ is a solution, then so is $v=-v(x)$. Incorporate this pairing behavior via the notation: $q_{ \pm}=q \pm v(x) / 2, p_{ \pm}=\nabla s\left(q_{ \pm}\right)$, $x_{ \pm}=\left(q_{ \pm}, p_{ \pm}\right)$. In the non-singular region where $\operatorname{det} \phi^{\prime \prime}(v(x), x) \neq 0$, the Wigner transform above has asymptotic form

$$
\rho_{0}(x ; \hbar) \approx h^{\frac{n}{2}}\left|\operatorname{det} \phi^{\prime \prime}(v(x), x)\right|^{-1 / 2} n\left(q_{+}\right) n\left(q_{-}\right) 2 \cos \left\{\frac{1}{\hbar} S_{0}(x)+\frac{\pi}{4} \operatorname{sgn} \phi^{\prime \prime}(v(x), x)\right\} .
$$

The phase $S_{0}(x)=\phi(v(x), x)$ and sgn denotes the signature of a symmetric matrix. If $x$ is in the neighborhood where (2.11) has no solutions, then $\rho_{0}(x)$ is $O\left(\hbar^{\infty}\right)$ small.

As Berry first noticed [9] (in the $n=1$ version of this problem), the phase $S_{0}$ is a symplectic area. To see this in the present context, define the $\chi_{1}$ Lagrangian manifold $\lambda \equiv\left\{x \in \chi_{1} \mid p=\nabla s(q)\right\}$. Construct two paths between the end points $x_{-}$and $x_{+}$. Let the first path, $C\left(x_{-}, x_{+}\right)$, be a straight line from $x_{-}$to $x_{+}$. As the second path, $\gamma_{\lambda}\left(x_{+}, x_{-}\right)$, choose any curve from $x_{+}$to $x_{-}$lying on the surface $\lambda$. Then one finds

$$
S_{0}(x)=\oint_{C\left(x_{-}, x_{+}\right)+\gamma_{\lambda}\left(x_{+}, x_{-}\right)} p \cdot d q .
$$

The geometry of the loop in (2.13) is the well known chord mid-point construct [10, 9, 21, 22, 23]. The end points $x_{ \pm}$of the directed chord $C\left(x_{-}, x_{+}\right)$lie on $\lambda$, and $x$ is the mid-point of this chord. By Stokes theorem, $S_{0}(x)$ is also the area of any membrane having boundary $C\left(x_{-}, x_{+}\right)+\gamma_{\lambda}\left(x_{+}, x_{-}\right)$.

\section{Symplectic Areas and WKB Phases}

This section shows how the secondary phase space WKB approximations for $U(t, x)$ or $\rho(t, x)$ can be reformulated in terms of primary phase space flows. This reduction process 
transforms all the $\chi_{2}$ WKB phases into symplectic areas defined by closed loops in $\chi_{1}$. A key element of this analysis is the solvability of the appropriate two point boundary condition (BC) problem. We pose the $\mathrm{BC}$ problem in terms of Lagrangian manifolds and construct both short and long time solutions.

To begin, we summarize Maslov's WKB expansion for the extended Schrödinger problem (2.1). The $t=0$ state for system (2.1) is assumed to have the generic form, $\Psi_{0}(x)=\alpha_{0}(x) e^{i \beta_{0}(x) / \hbar}$. The real functions $\alpha_{0}, \beta_{0}$ have support on the domain $D_{0} \subseteq \mathbb{R}_{x}^{2 n}$. The phase $\beta_{0}$ defines a $\chi_{2}$ Lagrangian manifold $\Lambda_{0}$, via $y(x)=\nabla \beta_{0}(x), x \in D_{0}$. Let $\Pi_{1}(x, y)=x$ be the projection onto $\mathbb{R}_{x}^{2 n}$. It is assumed that projection $\Pi_{1}: \Lambda_{0} \rightarrow D_{0}$ is a diffeomorphism.

To proceed three assumptions are required: (a) The function $\mathcal{H} \in T_{+}^{m}\left(\mathbb{R}_{z}^{4 n}\right), m \geq 2$. cf. Appendix A. (b) For arbitrary finite time intervals $[-T, T]$, the SPS Hamiltonian system (2.4) has unique smooth solutions, i.e. $G(t \mid x, y) \in C^{\infty}\left([-T, T], \mathbb{R}_{z}^{4 n}\right)$. (c) Let $x$ be a non-focal point ( $c f$. Definition 3) with respect to the $\Pi_{1}$ projection of the manifold $\Lambda_{t}=G(t) \Lambda_{0}$. Assume there are a finite number of points $x_{0}^{j} \in D_{0}, j=1, \ldots, N$ such that $\Pi_{1} G\left(t \mid x_{0}^{j}, \nabla \beta_{0}\left(x_{0}^{j}\right)\right)=x$.

Each of these assumptions is required for an evident reason: (a) ensures that $\mathcal{H}(\stackrel{2}{X}, \stackrel{1}{Y})$ is a well defined $\Psi \mathrm{DO}$, (b) prohibits finite time runaway trajectories, and (c) assumes the existence of one or more solutions to the BC problem: which trajectories starting from $\Lambda_{0}$ have $x$ as their final coordinate position? Locally varying $(t, x)$ in $(\mathrm{c})$ determines the initial $x_{0}$ as a function of $(t, x)$, namely $x_{0}^{j}=x_{0}^{j}(t, x)$.

Under the hypotheses $(\mathrm{a}-\mathrm{c})$ the $\hbar \rightarrow 0$ asymptotic solution of the Cauchy problem (2.1), with initial data $\Psi_{0}$, is

$$
\begin{aligned}
\Psi(t, x) & =\Psi^{s c}(t, x)[1+O(\hbar)] \\
\Psi^{s c}(t, x) & =\sum_{j=1}^{N} \frac{\phi_{j}(t, x)}{\sqrt{ }\left|J_{j}(t, x)\right|} \exp \left\{\frac{i}{\hbar} \beta_{j}(t, x)-\frac{i \pi}{2} m_{j}(t, x)\right\}, \quad t \in[-T, T] .
\end{aligned}
$$

Here $m_{j}(t, x)$ is the Maslov index for trajectory $\left\{G\left(\tau \mid z_{0}^{j}\right): \tau \in[0, t]\right\}$, where $z_{0}^{j}=$ $\left(x_{0}^{j}(t, x), \nabla \beta_{0}\left(x_{0}^{j}(t, x)\right)\right) \in \Lambda_{0}$. The phase and amplitude functions are

$$
\begin{aligned}
& \beta_{j}(t, x)=\beta_{0}\left(x_{0}^{j}\right)+\int_{0}^{t}\left[y\left(\tau \mid z_{0}^{j}\right) \cdot \dot{x}\left(\tau \mid z_{0}^{j}\right)-\mathcal{H}\left(G\left(\tau \mid z_{0}^{j}\right)\right)\right] d \tau \\
& J_{j}(t, x)=\left.\operatorname{det}\left(\frac{d x\left(t \mid x_{0}^{j}, \nabla \beta_{0}\left(x_{0}^{j}\right)\right)}{d x_{0}^{j}}\right)\right|_{x_{0}^{j}=x_{0}^{j}(t, x)}, \\
& \phi_{j}(t, x)=\left(\exp \int_{0}^{t} \operatorname{tr} \nabla_{x} \nabla_{y} \mathcal{H}\left(G\left(\tau \mid z_{0}^{j}\right)\right) d \tau\right) \alpha_{0}\left(x_{0}^{j}\right) .
\end{aligned}
$$

The family of operators $\mathcal{H}(\stackrel{2}{X}, \stackrel{1}{Y})$ consistent with the class $T_{+}^{m}\left(\mathbb{R}_{z}^{4 n}\right)$ is large. The growth index, $m$, must be 2 or greater so as to include kinetic energy in $H$. The operators $\mathcal{H}(\stackrel{2}{X}, \stackrel{1}{Y})$ need not have a polynomial dependence in momentum $Y$, nor do they need to be partial differential operators. This generality is essential to the SPS method we employ: normally, the $\Psi \mathrm{DO}$ defined by $\mathcal{H}_{1}$ and $\mathcal{H}_{2}$ will not be a finite order partial differential 
operator. The phases $\beta_{j}(t, x)$ have a geometric meaning. Define the $\mathcal{H}$ flow transported Lagrangian manifold, $\Lambda_{t} \equiv G(t) \Lambda_{0}$. Then the surfaces $y_{j}(t, x)=\nabla \beta_{j}(t, x)$ describe the different sheets of $\Lambda_{t}$. Representation (3.1 3.5) is Theorem 10.5 of [7].

In phase space quantum mechanics, the range of physical systems one may describe is controlled by the allowed functional form of the Weyl symbol Hamiltonian. For reasons of notational simplicity we presume $H$ is static. Including time dependent Hamiltonians, as a subsequent generalization, is a straightforward matter. The WKB summary above indicates that two restrictions on $H$ are necessary.

Assumption A1. The Hamiltonian operator $\widehat{H}$ is semiclassically admissible, specifically $H \in T_{+}^{m}\left(\mathbb{R}_{x}^{2 n}\right), m \geq 2$. A2. On finite time intervals $[-T, T], H$ generates unique classical flow, $g(t \mid x) \in C^{\infty}\left([-T, T], \mathbb{R}_{x}^{2 n}\right)$.

Assumption $\mathbf{A}$ is common to all the Propositions and Theorems of this section and will not be explicitly cited in them.

Since $l$ and $r$ are linear functions of $z$, Assumption A1 implies that the composite functions $\mathcal{H}_{1}=H \circ l$ and $\mathcal{H}_{2}=H \circ l-H \circ r$ are in $T_{+}^{m}\left(\mathbb{R}_{z}^{4 n}\right), m \geq 2$. Likewise, Lemma 2 shows that Assumption A2 guarantees that $G(t \mid x, y) \in C^{\infty}\left([-T, T], \mathbb{R}_{z}^{4 n}\right)$ for both $\mathcal{H}_{1}$ and $\mathcal{H}_{2}$ flow. The requirement that $H \in T_{+}^{m}\left(\mathbb{R}_{x}^{2 n}\right)(c f$. Appendix A) means that $H=H(x ; \hbar)$ has the $\hbar \rightarrow 0$ uniform asymptotic expansion

$$
H(x ; \hbar)=H_{0}(x)+\sum_{j=1}^{J} \hbar^{j} H_{j}(x)+O\left(\hbar^{J+1}\right) .
$$

For many physical systems $H(x ; \hbar)=H_{0}(x)$. For example, this occurs for a mass $m$ charged particle moving in an external electromagnetic field. The higher order terms $H_{j}(x)$ have no effect on the leading order WKB approximation. So from here on we will identify $H$ with $H_{0}$ and omit the 0 subscript.

\subsection{The Boundary Condition Problem}

The assumption A says nothing about the solvability of the BC problem. Rather than dealing with this by assumption, as was done in the representation (3.1 3.5), we will find existence proofs and explicit formulas for the BC solutions.

We discuss the $\mathrm{BC}$ conditions for the evolutions $U(t, x)$ and $\rho(t, x)$ in tandem. For the $U(t, x)$ evolution, take the initial state of the system to be

$$
U_{0}(x)=N_{0}(x) e^{i \Phi_{0}(x) / \hbar}, \quad x \in D_{0} \subseteq \mathbb{R}^{2 n}
$$

where the domain, $D_{0}$, is a simply connected open set. The phase $\Phi_{0}$ defines the single sheeted Lagrangian manifold $\Lambda_{0}=\left\{z \in \chi_{2} \mid y(x)=\nabla \Phi_{0}(x), x \in D_{0}\right\}$. The initial state for density matrix evolution $\rho(t, x)$ is presumed to be

$$
\rho_{0}(x)=\alpha_{0}(x) e^{i S_{0}(x) / \hbar}, \quad x \in D_{0} \subseteq \mathbb{R}^{2 n} .
$$

Again, $\Lambda_{0}$ will denote the simply connected manifold generated by $S_{0}$. The phases $\Phi_{0}$ and $S_{0}$ are both assumed to be $C^{\infty}\left(\mathbb{R}^{2 n}\right)$. 
BC Problem. Determine the trajectories $G\left(t \mid x_{0}, y_{0}\right)$ which begin on $\Lambda_{0}$ and have the final position $x \in D_{t}=\Pi_{1} \Lambda_{t}$.

(i) The Schrödinger case: Find the functions $x_{0}=x_{0}(t, x)$ and $y_{0}=y_{0}(t, x)$ from

$$
\begin{gathered}
\frac{1}{2}\left[g\left(t \mid x_{0}-\frac{1}{2} J y_{0}\right)+\left(x_{0}+\frac{1}{2} J y_{0}\right)\right]=x, \\
y_{0}=\nabla \Phi_{0}\left(x_{0}\right), \quad x_{0} \in D_{0} .
\end{gathered}
$$

(ii) The Heisenberg case: Find the functions $x_{0}=x_{0}(t, x)$ and $y_{0}=y_{0}(t, x)$ from

$$
\begin{gathered}
\frac{1}{2}\left[g\left(t \mid x_{0}-\frac{1}{2} J y_{0}\right)+g\left(t \mid x_{0}+\frac{1}{2} J y_{0}\right)\right]=x, \\
y_{0}=\nabla S_{0}\left(x_{0}\right), \quad x_{0} \in D_{0} .
\end{gathered}
$$

Lemma 2 justifies writing the left sides of (3.9) and (3.11) as the weighted sum of the left and right flows. It is natural to interpret each of these equations as a midpoint condition. Conditions (3.10) and (3.12) ensure that $\left(x_{0}, y_{0}\right)$ lies on $\Lambda_{0}$. Observe that the initial density matrix (3.8) is not real valued like approximation (2.12). However, by superposition with the complex conjugate of (3.8), the initial state can be made real.

We treat the small time and big time solutions to the $\mathrm{BC}$ problem with different methods and assumptions. The small time problem may be formulated as follows. Notice that the midpoint and $\Lambda_{0}$ boundary conditions may be combined into a single statement, $\mathcal{M}_{t}\left(x^{\prime}\right)=x$.

Definition 1. (i) In the Schrödinger problem set

$$
\mathcal{M}_{t}\left(x^{\prime}\right)=\widetilde{M}_{t}\left(x^{\prime}\right) \equiv \frac{1}{2}\left[g\left(t \mid x^{\prime}-\frac{1}{2} J \nabla \Phi_{0}\left(x^{\prime}\right)\right)+\left(x^{\prime}+\frac{1}{2} J \nabla \Phi_{0}\left(x^{\prime}\right)\right)\right]=x .
$$

(ii) In the Heisenberg problem set

$$
\mathcal{M}_{t}\left(x^{\prime}\right)=M_{t}\left(x^{\prime}\right) \equiv \frac{1}{2}\left[g\left(t \mid x^{\prime}-\frac{1}{2} J \nabla S_{0}\left(x^{\prime}\right)\right)+g\left(t \mid x^{\prime}+\frac{1}{2} J \nabla S_{0}\left(x^{\prime}\right)\right)\right]=x .
$$

The initial phase $\beta_{0}(x)$ in the extended Schrödinger problem is equal to either $\Phi_{0}(x)$ or $S_{0}(x)$ depending on whether one has case (i) or (ii). Here it is assumed that the support of $\beta_{0}(x)$ is all of $\mathbb{R}^{2 n}$, and that $x$ is any point in $\mathbb{R}^{2 n}$. If the non-linear function $\mathcal{M}_{t}$ is invertible, then the $\mathrm{BC}$ problem has a single trajectory specified by the initial values, $x_{0}(t, x)=\mathcal{M}_{t}^{-1}(x)$ and $y_{0}(t, x)=\nabla \beta_{0}\left(x_{0}(t, x)\right)$. The arguments of the flows $g(t)$ are the left-right projections of $\Lambda_{0}$.

Proposition 1. Let the Hessians $H^{\prime \prime}(x)$ and $\beta_{0}^{\prime \prime}(x)$ have $x$-uniform bounds

$$
\left\|H^{\prime \prime}(x)\right\| \leq c_{1}<\infty, \quad\left\|\beta_{0}^{\prime \prime}\left(x^{\prime}\right)\right\| \leq 2,
$$

then there exists a $t_{1}>0$ such that the midpoint map $\mathcal{M}_{t}: \mathbb{R}^{2 n} \rightarrow \mathbb{R}^{2 n}$ is a diffeomorphism for $t \in\left[-t_{1}, t_{1}\right]$. Specifically, $x_{0}(t, x)=\mathcal{M}_{t}^{-1}(x)$ is a unique $C^{1}\left(\left[-t_{1}, t_{1}\right], \mathbb{R}^{2 n}\right)$ solution of the $B C$ problems (i) and (ii), respectively.

Proof. Consider the Heisenberg problem. For parameters $(t, x) \in\left([-T, T], \mathbb{R}^{2 n}\right)$, define the family of maps $T_{t, x}: \mathbb{R}^{2 n} \rightarrow \mathbb{R}^{2 n}$,

$$
T_{t, x}\left(x^{\prime}\right) \equiv x+x^{\prime}-M_{t}\left(x^{\prime}\right)
$$


The idea of the proof is to show that $T_{t, x}$ is a contraction mapping. Its unique fixed point, $x^{*}(t, x)$, obeys $x=M_{t}\left(x^{*}(t, x)\right)$ and establishes that $M_{t}$ is invertible. Connect an arbitrary pair of points $\left(x_{1}, x_{2}\right)$ by the linear path $x(\xi)=x_{1}+\xi\left(x_{2}-x_{1}\right)$. Integrate $d T_{t, x}(x(\xi)) / d \xi$ on $\xi \in[0,1]$ to show

$$
\left|T_{t, x}\left(x_{2}\right)-T_{t, x}\left(x_{1}\right)\right| \leq\left|x_{2}-x_{1}\right| \int_{0}^{1}\left\|\nabla T_{t, x}(x(\xi))\right\| d \xi .
$$

Whenever the integral above is less than one for all $\left(x_{1}, x_{2}\right)$, then $T_{t, x}$ is a contraction mapping. Taking the $x^{\prime}$ gradient of $T_{t, x}\left(x^{\prime}\right)$ it follows that

$$
\begin{aligned}
\left\|\nabla T_{t, x}\left(x^{\prime}\right)\right\| & \leq \frac{1}{2}\left[\left\|I-\nabla g\left(t \mid l^{\prime}\right)\right\|+\left\|I-\nabla g\left(t \mid r^{\prime}\right)\right\|+\frac{1}{2}\left\|\nabla g\left(t \mid l^{\prime}\right)-\nabla g\left(t \mid r^{\prime}\right)\right\|\left\|S_{0}^{\prime \prime}\left(x^{\prime}\right)\right\|\right] \\
& <2\left(\exp c_{1}|t|-1\right) .
\end{aligned}
$$

To obtain the final inequality, $\left\|S_{0}^{\prime \prime}\left(x^{\prime}\right)\right\|$ was replaced by its bound 2 , and the Lemma 9 (Appendix A) estimates of the Jacobi field $\nabla g$ were employed. Let $t_{1}>0$ satisfy $2\left(\exp \left(c_{1} t_{1}\right)-1\right)<1$, then for times $|t| \leq t_{1}$ the rightmost combination above is less than one and $T_{t, x}$ is a contraction mapping for $(t, x) \in\left[-t_{1}, t_{1}\right] \times \mathbb{R}^{2 n}$. An application of the implicit function theorem to $M_{t}\left(x_{0}(t, x)\right)=x$ establishes that $x_{0} \in C^{1}\left(\left[-t_{1}, t_{1}\right], \mathbb{R}^{2 n}\right)$. The same argument applies to the Schrödinger BC problem.

The merit of the short time BC solution given in Proposition 1 is that it is global applicable for all $(t, x) \in\left[-t_{1}, t_{1}\right] \times \mathbb{R}^{2 n}$, and so in this time interval $\Lambda_{t}$ has a diffeomorphic $x$-projection. Nevertheless, the results are restrictive. Typically only a small time interval $\left[-t_{1}, t_{1}\right]$ is allowed, and the Hamiltonian and initial phase must have no more than quadratic growth as $|x| \rightarrow \infty$.

The $\mathrm{BC}$ problem in the large time regime has a different geometry. Let $\Lambda_{0}$ have an $x$-projection, $D_{0}$, that is contained within some compact set of $\mathbb{R}^{2 n}$. As $\Lambda_{t}$ evolves away from $\Lambda_{0}$, it may develop into a multi-sheeted manifold. Here, it is helpful to recall some of the terminology related to caustic behavior of Lagrangian manifolds, $\Lambda \subseteq \chi_{2}$.

Definition 2. A point $m \in \Lambda$ is non-singular if it has a neighborhood that is diffeomorphically projected onto $\mathbb{R}_{x}^{2 n}$. If the opposite holds, it is singular. The set $\Sigma(\Lambda)$, of all singular points, is called the cycle of singularities. Its complement is the regular set, $\mathcal{R}=\Lambda / \Sigma(\Lambda)$.

Definition 3. The $x$ projection of the cycle of singularities is the caustic or focal set, $\Pi_{1}(\Sigma(\Lambda))=\mathcal{K}(\Lambda)$. Let $D_{t}=\Pi_{1} \Lambda_{t}$ denote the $x$-image of $\Lambda_{t}$, and $\mathcal{K}\left(\Lambda_{t}\right)$ the associated caustic set. The non-focal set is defined as the complement of the caustic set, $\mathcal{N}_{t}=$ $D_{t} / \mathcal{K}\left(\Lambda_{t}\right)$.

If $\Lambda_{t}$ has more than one sheet, the inverse of projection $\Pi_{1}: \Lambda_{t} \rightarrow D_{t}$ ceases to be single valued: there are $x \in \mathcal{N}_{t}$ with multiple pre-images, $\left\{m^{j}\right\}_{1}^{N} \subseteq \Lambda_{t}$, satisfying $\Pi_{1} m^{j}=x$. The result below is stated for the Heisenberg BC problem, with obvious adjustment it applies to the Schrödinger problem. 
Proposition 2. Let $\tilde{x} \in \mathcal{N}_{t} \subseteq D_{t}$ be a non-focal point with pre-images $\left\{m^{j} \in \Lambda_{t}\right\}_{1}^{N}$. Then the Heisenberg $B C$ problem has solutions

$$
M_{t}\left(x_{0}^{j}(t, x)\right)=x, \quad j=1, \cdots, N .
$$

The functions, $x_{0}^{j}(t, x)$, are smooth and have support in a neighborhood, $\mathcal{N}_{t} \subseteq \widetilde{U}_{j} \ni \tilde{x}$.

Proof. Let $F_{t}\left(x^{\prime}, x\right)=M_{t}\left(x^{\prime}\right)-x$, where $F_{t}$ is smooth on the domain $\left(x^{\prime}, x\right) \in D_{0} \times$ $\mathcal{N}_{t}$. The implicit function theorem (IFT) solutions generated from $F_{t}\left(x^{\prime}, x\right)=0$, will construct $x_{0}^{j}(t, x)$. In order to apply the IFT one must have a solution pair $\left(x_{0}, x\right)$, where $F_{t}\left(x_{0}, x\right)=0$ and det $\nabla_{x^{\prime}} F_{t}\left(x_{0}, x\right) \neq 0$. Consider one pre-image $m^{j}$ of $\tilde{x}$. Each $m^{j} \in \Lambda_{t}$ has a 1-1 association with a point in $D_{0}$, via $x_{0}=\Pi_{1} G\left(-t \mid m^{j}\right)$. The set $\left(x_{0}, \tilde{x}\right)$ is a solution pair of $F_{t}$. It remains to show that the determinant is non-zero. Since $\tilde{x} \in \mathcal{N}_{t}$, Lemma 10 implies that $\operatorname{det} \nabla M_{t}\left(x_{0}\right) \neq 0$. Furthermore $\nabla_{x^{\prime}} F_{t}\left(x^{\prime}, x\right)=\nabla M_{t}\left(x^{\prime}\right)$ shows that det $\nabla_{x^{\prime}} F_{t}\left(x_{0}, \tilde{x}\right) \neq 0$. The IFT then establishes the existence of $x_{0}^{j}(t, x)$ defined on a neighborhood, $\widetilde{U}_{j} \ni \tilde{x}$. If $F_{t} \in C^{p}, p \geq 1$, then $x_{0}^{j} \in C^{p}$.

Once $\Lambda_{t}$ has become multi-sheeted, typically the regular set $\mathcal{R}_{t}$ is divided by the cycle of singularities into disjoint subsets $\left\{\Lambda_{t}^{j}\right\}_{1}^{N}$. On $\Lambda_{t}^{j} \subset \mathcal{R}_{t}$, the restricted projection $\Pi_{1}^{j} \Lambda_{t}^{j} \equiv$ $D_{t}^{j} \subset \mathcal{N}_{t}$ is diffeomorphic. In this circumstance, $x_{0}^{j}(t, x)$ has the explicit representation

$$
x_{0}^{j}(t, x)=\Pi_{1} G\left(-t \mid\left(\Pi_{1}^{j}\right)^{-1} x\right), \quad x \in D_{t}^{j} .
$$

Identity (3.16) determines $x_{0}^{j}(t, x)$ directly without requiring an IFT construction.

As is evident from $J(x, t)=\operatorname{det} \nabla \mathcal{M}_{t}(x), c f$. (3.4), the gradient structures in the BC problem determine the WKB amplitudes as functions of the left and right Jacobi fields, $\nabla g\left(t \mid l^{\prime}\right)$ and $\nabla g\left(t \mid r^{\prime}\right)$. It is of interest to find a more geometric representation of these amplitudes. This is achieved by constructing a suitable $\chi_{2}$ Poisson bracket related to the BC problem. First characterize the initial manifold $\Lambda_{0}$ by the (independent) constraint functions

$$
\zeta\left(x^{\prime}, y^{\prime}\right)=y^{\prime}-\nabla \Phi_{0}\left(x^{\prime}\right), \quad \eta\left(x^{\prime}, y^{\prime}\right)=y^{\prime}-\nabla S_{0}\left(x^{\prime}\right) .
$$

The conditions $\zeta\left(x^{\prime}, y^{\prime}\right)=0$ and $\eta\left(x^{\prime}, y^{\prime}\right)=0$ define $\Lambda_{0}$ in our two problems. A second set of functions must necessarily carry information about the $\mathcal{H}$ dynamics. Select the midpoint flow functions $x\left(t \mid x^{\prime}, y^{\prime}\right)$ for this role. Then one finds

Lemma 3. In terms of the $\Lambda_{0}$ constraint functions, $\zeta$ and $\eta$, the $\mathcal{H}_{1}$ and $\mathcal{H}_{2}$ amplitudes have the $\chi_{2}$ Poisson bracket representation

$$
\begin{aligned}
\operatorname{det} \nabla \widetilde{M}_{t}\left(x^{\prime}\right) & =\operatorname{det}\{x(t), \zeta\}_{2}\left(x^{\prime}, \nabla \Phi_{0}\left(x^{\prime}\right)\right) . \\
\operatorname{det} \nabla M_{t}\left(x^{\prime}\right) & =\operatorname{det}\{x(t), \eta\}_{2}\left(x^{\prime}, \nabla S_{0}\left(x^{\prime}\right)\right) .
\end{aligned}
$$

Proof. Consider the $\mathcal{H}_{2}$ case, an elementary calculation shows that

$$
\begin{aligned}
\left\{x(t)_{\alpha}, \eta_{\beta}\right\}_{2}\left(x^{\prime}, \nabla S_{0}\left(x^{\prime}\right)\right) & =\frac{1}{2}\left[\nabla g\left(t \mid l^{\prime}\right)\left(I-\frac{1}{2} J S_{0}^{\prime \prime}\left(x^{\prime}\right)\right)+\nabla g\left(t \mid r^{\prime}\right)\left(I+\frac{1}{2} J S_{0}^{\prime \prime}\left(x^{\prime}\right)\right)\right] \\
& =\left[\nabla M_{t}\left(x^{\prime}\right)\right]_{\alpha \beta} .
\end{aligned}
$$


The determinant of this matrix is (3.18). The same type of argument gives (3.17).

This type of bracket representation of the amplitudes is common [19, 22 in the SPS context. The components of constraint $\zeta$ (and $\eta$ ) are commutative with respect the $\chi_{2}$ bracket. This involution property is a necessary and sufficient condition [24] for the manifold $\Lambda_{0}$ to be Lagrangian. In addition, the midpoint variables $x(t)$ are commutative. This is a result of the fact that the functions $x(t)$ take their values on PPS plane which is $\chi_{2}$ Lagrangian.

Our boundary condition analysis is concluded by identifying a $\chi_{1}$ loop structure that is created by each $\mathrm{BC}$ solution. These loops are constructed from two types of $\chi_{1}$ line segments - chords and trajectories. The quantity $C\left(x_{1}, x_{2}\right)$ denotes the chord (the $\mathbb{R}^{2 n}$ geodesic) from $x_{1}$ to $x_{2}$. Trajectory type line segments are specified by

$$
\begin{aligned}
& T^{+}\left(x_{1}, x_{2}\right) \equiv\left\{g\left(\tau \mid x_{1}\right) ;(\tau: 0 \rightarrow t)\right\}, \quad x_{2}=g\left(t \mid x_{1}\right), \\
& T^{-}\left(x_{1}, x_{2}\right) \equiv\left\{g\left(\tau \mid x_{1}\right) ;(\tau: 0 \rightarrow-t)\right\}, \quad x_{2}=g\left(-t \mid x_{1}\right) .
\end{aligned}
$$

The \pm labelling distinguishes between forward and backward evolution.

Consider the Schrödinger problem with mid-point BC solution $x_{0}(x, t)$. The $\Lambda_{0}$ manifold requirement (3.10), when stated in the left-right variables, is

$$
l_{0}(t, x)=x_{0}(t, x)-\frac{1}{2} \nabla \Phi_{0}\left(x_{0}(t, x)\right), \quad r_{0}(t, x)=x_{0}(t, x)+\frac{1}{2} \nabla \Phi_{0}\left(x_{0}(t, x)\right) .
$$

After time displacement $t$, the $\mathcal{H}_{1}$ flow moves $l_{0}=l_{0}(t, x)$ and $r_{0}=r_{0}(t, x)$ into the new positions $l_{t}=l_{t}(t, x)=g\left(t \mid l_{0}\right)$ and $r_{t}=r_{t}(t, x)=r_{0}, c f$. Lemma 2(i).

The pair of points $\left(l_{t}, r_{0}\right)$ define the chord $C\left(l_{t}, r_{0}\right)$, having midpoint $x$. However, $\left(l_{t}, r_{0}\right)$ are also the endpoints of the linked line segments, $C\left(r_{0}, l_{0}\right)+T^{+}\left(l_{0}, l_{t}\right)$. Altogether, for each $x \in \mathcal{N}_{t}$, one has (see Fig. 1) a three sided oriented loop

$$
\widetilde{L}(t, x)=C\left(r_{0}, l_{0}\right)+T^{+}\left(l_{0}, l_{t}\right)+C\left(l_{t}, r_{0}\right) .
$$

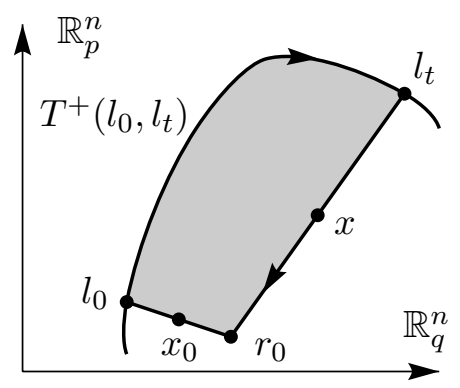

Figure 1. The Schrödinger loop.

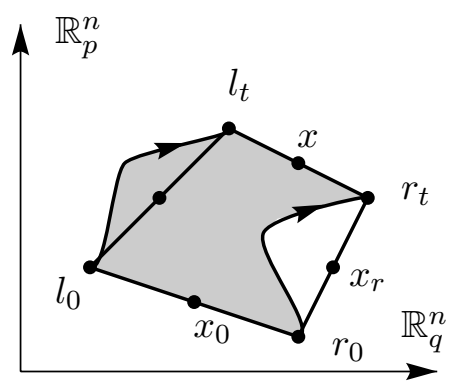

Figure 2. The Heisenberg BC solution.

\subsection{Evolution Representations}

Based on these solutions of the BC problem the WKB approximation for $U(t, x)$ takes the following form. 
Theorem 1. Suppose $x$ is a non-focal point associated with the manifold $\Lambda_{t}$. Let $\left\{\widetilde{L}^{j}(t, x)\right\}_{1}^{N}$ be the family of loops generated by the Schrödinger BC solutions $\left\{x_{0}^{j}(t, x)\right\}_{1}^{N}, x \in \mathcal{N}_{t}$. Then the $O(\hbar)$ approximation of $U(t, x)$ is

$$
\begin{aligned}
U^{s c}(t, x) & =\sum_{j=1}^{N} N_{j}(t, x) \exp \left\{\frac{i}{\hbar} \Phi_{j}(t, x)-\frac{i \pi}{2} m_{j}(t, x)\right\}, \\
\Phi_{j}(t, x) & =\Phi_{0}\left(x_{0}^{j}(t, x)\right)+\oint_{\widetilde{L}^{j}(t, x)} p \cdot d q-H\left(l_{0}^{j}(t, x)\right) t, \\
N_{j}(t, x) & =\left|\operatorname{det} \nabla \widetilde{M}_{t}\left(x_{0}^{j}(t, x)\right)\right|^{-1 / 2} N_{0}\left(x_{0}^{j}(t, x)\right) .
\end{aligned}
$$

Before proceeding to the Heisenberg case, it is useful to make a number of remarks specific to Theorem 1. An important special case occurs in the problem where $U(0, x)=1$. Here $\Phi_{0} \equiv 0$ and $\Lambda_{0} \subseteq \mathbb{R}_{x}^{2 n}$. In particular, $l_{0}=x_{0}=r_{0}$, so the loop $\widetilde{L}^{j}(t, x)$ simplifies into one chord $C\left(l_{t}, l_{0}\right)$ with midpoint $x$ and one dynamical curve $T^{+}\left(l_{0}, l_{t}\right)$ connecting the chord end points. If $\Lambda_{t}$ has one sheet, then $N=1$, and the theorem above gives the representation obtained in [10]. However, it should be noted that Marinov did not investigate the $\mathrm{BC}$ problem. Instead his approach was to construct the phase $\Phi=\Phi_{1}(t, x)$ by solving the Hamilton-Jacobi $(\mathrm{H}-\mathrm{J})$ equation associated [10, 8, 25] with the evolution problem (1.1),

$$
\partial_{t} \Phi(t, x)-H\left(x-\frac{1}{2} J \nabla \Phi(t, x)\right)=0
$$

with the Cauchy initial data $\Phi(0, x)=0$. Because of the highly non-linear character of $H(x)$ this $\mathrm{H}-\mathrm{J}$ problem is much more difficult to solve than the $\mathrm{BC}$ problem (3.13) treated above. It is straight forward to verify that $\Phi(t, x)$ given by (3.21) is a solution of (3.23).

Marinov's work established the central role of the phase $\Phi(t, x)$ in the semiclassical approximation of $U(t, x)$. However, knowledge of the $\mathrm{H}-\mathrm{J}$ equation in the form (3.23), and the use of $\Phi(t, x)$ as the generator of a canonical transformation, $c f$. (2.8), goes back to Poincaré [26].

Quantum operator identities induce corresponding Weyl symbol identities. A simple example of this is the unitarity property, $U(t)=U(-t)^{\dagger}$. Its Weyl symbol image is $U(t, x)=U(-t, x)^{*}$, which in turn requires that $\Phi(t, x)=-\Phi(-t, x)$ and $N(t, x)=$ $N(-t, x)$.

The generalization of Theorem 1 to non-static Hamiltonians $H(t, x)$ is achieved if the phase $\Phi_{j}(t, x)$ is modified by the replacement

$$
H\left(l_{0}^{j}(t, x)\right) t \rightsquigarrow \int_{0}^{t} H\left(g\left(\tau \mid l_{0}^{j}(t, x)\right) d \tau .\right.
$$

Adjusting the notation above to cover the Heisenberg problem is easy. In this case $l_{0}(t, x)$ and $r_{0}(t, x)$ are defined by (3.19) with $S_{0}$ replacing $\Phi_{0}$. The right point $r_{t}$ is no longer the fixed point $r_{0}$, but instead is $r_{t}=r_{t}(t, x)=g\left(t \mid r_{0}(t, x)\right)$. The curve between $r_{t}$ and $r_{0}$ is $T^{-}\left(r_{t}, r_{0}\right)$. The resultant $\mathrm{BC}$ loop is the four segment structure (see Fig. 2)

$$
W(t, x) \equiv C\left(r_{0}, l_{0}\right)+T^{+}\left(l_{0}, l_{t}\right)+C\left(l_{t}, r_{t}\right)+T^{-}\left(r_{t}, r_{0}\right) .
$$


The loop $W(t, x)$ is like the Schrödinger BC loop $\widetilde{L}(t, x)$, but has an additional side $T^{-}\left(r_{t}, r_{0}\right)$ inserted at the point $r_{0}$. However, there is another simpler loop generated by the BC solution. Denote by $\gamma\left(t \mid C\left(r_{0}, l_{0}\right)\right) \equiv\left\{g\left(t \mid x^{\prime}\right): x^{\prime} \in C\left(r_{0}, l_{0}\right)\right\}$. This curve is the flow translated image of the chord $C\left(r_{0}, l_{0}\right)$. In general it is no longer a straight line but it has end points $\left(r_{t}, l_{t}\right)$, so

$$
L(t, x) \equiv C\left(l_{t}, r_{t}\right)+\gamma\left(t \mid C\left(r_{0}, l_{0}\right)\right)
$$

defines a two segment closed curve, see Fig. 3. We refer to $L(t, x)$ as the Heisenberg loop.

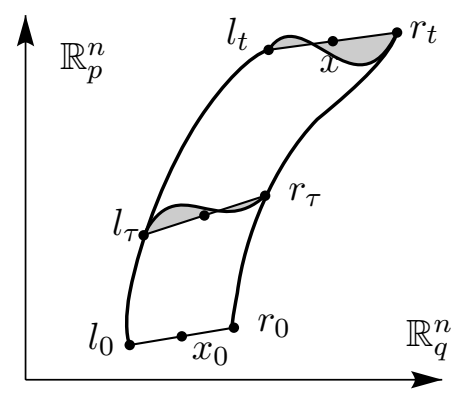

Figure 3. Heisenberg loop area $L(t, x)$.

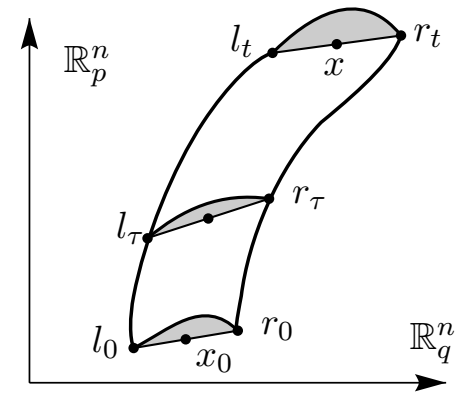

Figure 4. $S(t, x)$ as an evolving front.

Theorem 2. Suppose $x$ is a non-focal point associated with the manifold $\Lambda_{t}$. Let $\left\{L^{j}(t, x)\right\}_{1}^{N}$ be the family of Heisenberg loops generated by the $B C$ solutions $\left\{x_{0}^{j}(t, x)\right\}_{1}^{N}, x \in \mathcal{N}_{t}$. Then the $O(\hbar)$ approximation of $\rho(t, x)$ is

$$
\begin{aligned}
\rho^{s c}(t, x) & =\sum_{j=1}^{N} \alpha_{j}(t, x) \exp \left\{\frac{i}{\hbar} S_{j}(t, x)-\frac{i \pi}{2} m_{j}(t, x)\right\}, \\
S_{j}(t, x) & =S_{0}\left(x_{0}^{j}(t, x)\right)+\oint_{L^{j}(t, x)} p \cdot d q, \\
\alpha_{j}(t, x) & =\left|\operatorname{det} \nabla M_{t}\left(x_{0}^{j}(t, x)\right)\right|^{-1 / 2} \alpha_{0}\left(x_{0}^{j}(t, x)\right) .
\end{aligned}
$$

Proof. Within the present setup, direct computation establishes both Theorems 1 and 2 . Consider the Heisenberg case. The $d \tau$ integral contribution to the $\chi_{2}$ WKB phase in (3.3) has both a $y \cdot \dot{x}$ and a $\mathcal{H}_{2}$ contribution. The first of these is

$$
\begin{aligned}
\int_{0}^{t} y(\tau) \cdot \dot{x}(\tau) d \tau & =\frac{1}{2} \int_{0}^{t}\left(\dot{g}\left(\tau \mid l_{0}\right)+\dot{g}\left(\tau \mid r_{0}\right)\right) \cdot J\left(g\left(\tau \mid l_{0}\right)-g\left(\tau \mid r_{0}\right)\right) d \tau \\
& =\oint_{W(t, x)} p \cdot d q .
\end{aligned}
$$

The top equality is the result of Lemma 2(ii). The lower equality follows from an integration by parts. For example

$$
\int_{0}^{t} \dot{g}\left(\tau \mid l_{0}\right) \cdot J g\left(\tau \mid l_{0}\right) d \tau=2 \int_{0}^{t} p\left(\tau \mid l_{0}\right) \cdot \dot{q}\left(\tau \mid l_{0}\right) d \tau+\left(q_{0}^{l} \cdot p_{0}^{l}-q_{t}^{l} \cdot p_{t}^{l}\right),
$$


where $\left(q_{0}^{l}, p_{0}^{l}\right)=l_{0}$ and $\left(q_{t}^{l}, p_{t}^{l}\right)=l_{t}$. The $d \tau$ integral is that of $p \cdot d q$ over the curve $T^{+}\left(l_{0}, l_{t}\right)$. The sum of all the surface terms from (3.27) is just the integral over the two chords, $C\left(r_{0}, l_{0}\right)+C\left(l_{t}, r_{t}\right)$. Thus one has that the time integral (3.27) is the loop integral (3.28)

The $\mathcal{H}_{2} d \tau$ term may be rewritten in loop form by employing the Poincaré-Cartan identity in Lemma 11. Setting the initial curve to be $\gamma_{0}=C\left(r_{0}, l_{0}\right)$, the identity (C.1) is

$$
\left(H\left(l_{0}\right)-H\left(r_{0}\right)\right) t=\oint_{C\left(r_{0}, l_{0}\right)+T^{+}\left(l_{0}, l_{t}\right)+g\left(t \mid C\left(l_{0}, r_{0}\right)\right)+T^{-}\left(r_{t}, r_{0}\right)} p \cdot d q .
$$

Adding all the loop integrals gives (3.25).

The amplitude expressions result from $M_{t}\left(x_{0}^{j}(t, x)\right)=x$, and the fact that the exponentiated integral in (3.5) vanishes. This latter is a consequence of $\operatorname{tr} \nabla_{x} \nabla_{y} H \circ l=$ $\operatorname{tr} H^{\prime \prime}\left(-\frac{1}{2} J\right)=0$, etc.

The Hamilton-Jacobi equation for the $\rho(t, x)$ problem is

$$
\partial_{t} S(t, x)-H\left(x-\frac{1}{2} J \nabla S(t, x)\right)+H\left(x+\frac{1}{2} J \nabla S(t, x)\right)=0 .
$$

All the $S_{j}(t, x)$ functions, provided by (3.25), are solutions of this Heisenberg H-J equation. As $t$ nears $0, \Lambda_{t}$ will have one sheet. Its phase obeys, $S(0, x)=S_{0}(x)$.

Lemma 10 establishes that the amplitudes $N_{j}(t, x)$ and $\alpha_{j}(t, x)$ are finite for all $x \in \mathcal{N}_{t}$. When $x \in \mathcal{K}\left(\Lambda_{t}\right)$ the amplitudes diverge and the representations of Theorem 1 and 2 are not applicable at these caustic points. If $x$ is not in $D_{t}$, it is classically forbidden. In this sector, $U(t, x)$ and $\rho(t, x)$ are $O\left(\hbar^{\infty}\right)$.

The area representation of $S(t, x)$ is a consequence of Stokes' theorem,

$$
S(t, x)=S_{0}\left(x_{0}(t, x)\right)+\int_{\sigma(L(t, x))} d q \wedge d p .
$$

Above, $\sigma(L(t, x))$ denotes any oriented surface with boundary $L(t, x)$. The specific form of (3.29) resulted form choosing the $\gamma_{0}$ curve to be $C\left(r_{0}, l_{0}\right)$. Other natural choices for the curve connecting $r_{0}$ and $l_{0}$ do occur. Consider the problem where $\rho_{0}(x)$ is given by the projection operator $\hat{\rho}_{0}=\left|\psi_{0}\right\rangle\left\langle\psi_{0}\right|$. As was noted in Sec. 2 the initial phase function $S_{0}(x)$ is the loop $C\left(l_{0}, r_{0}\right)+\gamma_{\lambda}\left(r_{0}, l_{0}\right)$ integral (2.13). By choosing $\gamma_{0}=\gamma_{\lambda}\left(r_{0}, l_{0}\right)$ in the identity (C.1) one can incorporate the initial phase into the final loop integral. So for this particular initial state, the entire Heisenberg phase has the form of a symplectic area

$$
S(t, x)=\int_{\sigma\left(L^{\prime}(t, x)\right)} d q \wedge d p
$$

where $L^{\prime}(t, x)=g\left(t \mid \gamma_{\lambda}\left(r_{0}, l_{0}\right)\right)+C\left(l_{t}, r_{t}\right)$.

The phases $\Phi(t, x)$ and $S(t, x)$ form representations of the $\chi_{2}$ Lagrangian manifolds $\Lambda_{t}$, via $y=\nabla \Phi(t, x)$ and $y=\nabla S(t, x)$. In turn these phases define, $c f$. (2.8), a canonical transformation between the dynamical left right coordinates $l\left(t \mid l_{0}\right)$ and $r\left(t \mid r_{0}\right)$. The $\Lambda_{t}$ phases also generate dynamical flow. In the Heisenberg case one has

$$
l\left(t \mid l_{0}\right)=g\left(t \mid l_{0}\right)=x-\frac{1}{2} J \nabla S(t, x), \quad r\left(t \mid r_{0}\right)=g\left(t \mid r_{0}\right)=x+\frac{1}{2} J \nabla S(t, x) .
$$


The presence of symmetries add structure and simplify these semiclassical expansions. Two Weyl symbol specific symmetries are: affine canonical covariance and $\hbar$-parity invariance.

Consider the first of these. In the notation of Appendix A, let $V$ be the unitary operator defined by the affine transformation $A: \chi_{1} \rightarrow \chi_{1}$. Let $U_{V}(t)$ and $\rho_{V}(t)$ be the symbols of the $V$-similarity transformed evolutions $\widehat{U}_{V}(t) \equiv V \widehat{U}(t) V^{-1}$ and $\hat{\rho}_{V}(t) \equiv$ $V \hat{\rho}(t) V^{-1}$. The semiclassical covariance statements are

$$
U_{V}^{s c}(t)=U^{s c}(t) \circ A^{-1}, \quad \rho_{V}^{s c}(t)=\rho^{s c}(t) \circ A^{-1}
$$

These identities follow from the exact covariance property, e.g. $\rho_{V}(t)=\rho(t) \circ A^{-1}$, and the fact that asymptotic expansions have unique coefficients.

The $\hbar$ parity property is specific to Heisenberg evolution. Recall that, $c f$. (2.9), the symbol $\rho_{0}(x ; \hbar)$ for the initial state $\hat{\rho}_{0}=\left|\psi_{0}\right\rangle\left\langle\psi_{0}\right|$ is an even function of $\hbar$. For most problems of physical interest this $\hbar$-even property is maintained under time evolution. Specifically, one has

Lemma 4. (Evolution $\hbar$-parity). Suppose the real valued $H(x ; \hbar)$ and $\rho_{0}(x ; \hbar)$ have even $\hbar$ parity. Then the solution of (1.耳), $\rho(t, x)$, is real and has even $\hbar$ parity. The associated semiclassical expansion has the improved convergence

$$
\begin{aligned}
\rho(t, x) & =\rho^{s c}(t, x)\left[1+O\left(\hbar^{2}\right)\right], \\
\rho^{s c}(t, x) & =\left|\operatorname{det} \nabla M_{t}\left(x_{0}(t, x)\right)\right|^{-1 / 2} \alpha_{0}\left(x_{0}(t, x)\right) \cos [S(t, x) / \hbar] .
\end{aligned}
$$

Proof. The function $\rho(t, x)=\rho(t, x ; \hbar)$ is defined as the unique solution of (1.2). Since the Moyal bracket and $H(x ; \hbar)$ are $\hbar$ even, the equation of motion (1.2) is invariant under $\hbar \rightarrow-\hbar$. Because initial state the $\rho_{0}(x ; \hbar)$ is even it follows that $\rho(t, x ; \hbar)$ is $\hbar$ even.

It is useful to review to what extent the use of the secondary phase approach is critical in obtaining Theorems 1 and 2. Marinov obtained the small time, $\Phi_{0}=0$, version of Theorem 1 without using the embedding of $\chi_{1}$ into $\chi_{2}$. In this approach (see [25] for related details) one starts with problem (1.1), and assumes a WKB representation for $U(t, x) \approx U^{s c}(t, x)$. After applying the definition of the $*$ product to $H * U^{s c}(t)$ the Hamilton-Jacobi equation equation (3.23) for $\Phi(t, x)$ is derived from the $\hbar \rightarrow 0$ limit. The transport equations for amplitudes $N(t, x)$ are obtained from (1.1) by extracting the ordinary differential equations generated by the higher order $\hbar$ term identities. A similar approach would work for the $\rho(t, x)$ problem. What this strictly primary phase space approach misses is the recognition of the Lagrangian manifold $\Lambda_{t}$ as the geometric structure that determines the Schrödinger and Heisenberg WKB expansions. Given the SPS setting one can make the WKB representations global by following Maslov's approach. In Theorems 1 and 2 the base Lagrangian plane was always taken to be $\mathbb{R}_{x}^{2 n}$ with commutative coordinates $\left\{x_{\alpha}\right\}_{1}^{2 n}$. The caustic and non-diffeomorphic behavior of the $\Pi_{1}$ projection is avoided by replacing $\Pi_{1}$ by a different and non-singular projection: $\Pi^{*} \Lambda_{t}=\Lambda^{*}$. Here $\Lambda^{*}$ is a a new Lagrangian plane with mixed (but commuting) coordinates $\left\{x_{\alpha(i)}, y_{\beta(i)}: i=1, \ldots, 2 n\right\}$. A related point concerns the amplitude representations. Only in the SPS setting is it possible to express the amplitudes as determinants of a Poisson bracket. 


\section{Applications and Examples}

In this section we collect various interpretations, extensions and applications of Theorems 1 and 2. For example, in both the Schrödinger and Heisenberg pictures, time evolution is implemented by a one parameter group. Define $\Gamma(t) \hat{\rho}_{0} \equiv U(t) \hat{\rho}_{0} U(t)^{\dagger}$. These two group properties are $U\left(t_{1}+t_{2}\right)=U\left(t_{1}\right) U\left(t_{2}\right)$ and $\Gamma\left(t_{1}+t_{2}\right)=\Gamma\left(t_{1}\right) \circ \Gamma\left(t_{2}\right)$, respectively. What are the symplectic phase identities generated by these groups? Another question of interest is to establish the mutual consistency of the $U^{s c}(t, x)$ and $\rho^{s c}(t, x)$ approximations.

The systems (1.1) and (1.2) admit exact solutions if the Hamiltonians are quadratic. In this special case, the contrasting behavior of $U(t, x)$ and $\rho(t, x)$ is explored. Finally, we show how the semi-classical solutions of (1.2) behave when $\rho_{0}(x)$ is not a rapidly oscillating function of $\hbar$.

\subsection{Phase Additivity Identities}

Phase additivity identities, mirroring the group properties of $U(t)$ and $\Gamma(t)$, are a direct consequence of the loop structure of the BC problem. Denote the BC loop integrals by

$$
\varphi(t, x ; \widetilde{L})=\oint_{\widetilde{L}(t, x)} p \cdot d q, \quad \mathcal{A}(t, x ; W)=\oint_{W(t, x)} p \cdot d q .
$$

First consider the Schrödinger problem. Let $t_{1}$ and $t_{2}$ be successive time displacements. For $x \in \mathcal{N}_{t_{1}+t_{2}}$ the BC solution determines, $x_{0}\left(t_{1}+t_{2}, x\right)$, (where the $j$ label is suppressed). The loop for the full time interval is

$$
\widetilde{L}_{1+2}\left(t_{1}+t_{2}, x\right) \equiv T^{+}\left(l_{0}, l_{1+2}\right)+C\left(l_{1+2}, r_{0}\right)+C\left(r_{0}, l_{0}\right) .
$$

Here $l_{1+2}=g\left(t_{1}+t_{2} \mid l_{0}\right)$. As Fig. 5 shows this loop can be decomposed into the sum of two (or more) sub-loops defined for $t_{1}$ and $t_{2}$, e.g. $l_{1}=g\left(t_{1} \mid l_{0}\right)$. Let $\widetilde{L}_{1}$ and $\widetilde{L}_{2}$ be the loops with endpoints $\left(l_{0}, l_{1}, r_{0}\right)$ and $\left(l_{1}, l_{1+2}, r_{0}\right)$. It follows from their definitions that these loops are additive,

$$
\widetilde{L}_{1+2}\left(t_{1}+t_{2}, x\right)=\widetilde{L}_{1}\left(t_{1}, x_{1}\right)+\widetilde{L}_{2}\left(t_{2}, x\right), \quad x_{1}=\frac{1}{2}\left(l_{1}+r_{0}\right) .
$$

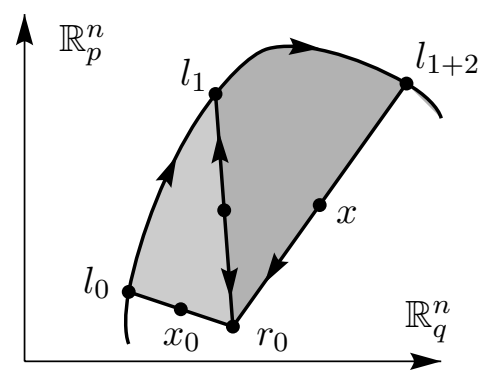

Figure 5. Schrödinger loop addition.

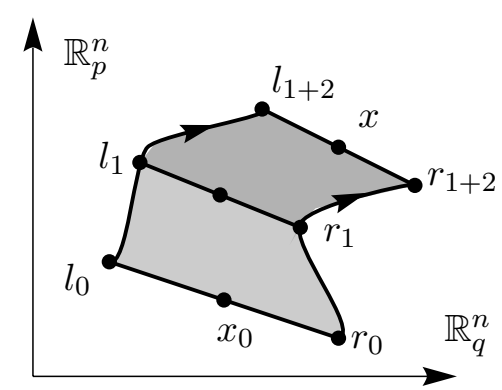

Figure 6 . Heisenberg loop addition.

As Fig. 6 makes clear there is a corresponding loop additivity in the Heisenberg BC problem. Note that there is no requirement that the intermediate midpoint $\left(t_{1}, x_{1}\right)$ be non-focal. Using the phase definitions above one obtains the following. 
Proposition 3. Let $x \in \mathcal{N}_{t_{1}+t_{2}}$ and suppose, $\widetilde{L}_{1+2}^{j}=\widetilde{L}_{1}^{j}+\widetilde{L}_{2}^{j} ; W_{1+2}^{j}=W_{1}^{j}+W_{2}^{j}$, are loop decompositions consistent with the $B C$ solutions $\widetilde{M}_{t_{1}+t_{2}}\left(x_{0}^{j}\left(t_{1}+t_{2}, x\right)\right)=x$ and $M_{t_{1}+t_{2}}\left(x_{0}^{j}\left(t_{1}+t_{2}, x\right)\right)=x, j=1, \ldots, N$, then

$$
\begin{aligned}
\varphi\left(t_{1}+t_{2}, x ; \widetilde{L}_{1+2}^{j}\right) & =\varphi\left(t_{1}, x_{1}^{j} ; \widetilde{L}_{1}^{j}\right)+\varphi\left(t_{2}, x ; \widetilde{L}_{2}^{j}\right), \\
\mathcal{A}\left(t_{1}+t_{2}, x ; W_{1+2}^{j}\right) & =\mathcal{A}\left(t_{1}, x_{1}^{j} ; W_{1}^{j}\right)+\mathcal{A}\left(t_{2}, x ; W_{2}^{j}\right) .
\end{aligned}
$$

Consider the short time regime where Proposition 1 holds. Let $\Phi_{0}=0$ and $x_{0}(\tau, x)=$ $\widetilde{M}_{\tau}^{-1}(x)$ be the BC solution. In this sector, it is possible to compare the rule (4.1) with the phase addition formula established by Marinov [10]. Denote by $X(\tau, x)$ the two segment loop $T^{+}\left(x_{0}, g\left(\tau \mid x_{0}\right)\right)+C\left(g\left(\tau \mid x_{0}\right), x_{0}\right)$, where $x_{0}=l_{0}=\widetilde{M}_{\tau}^{-1}(x)$. Suppose $\varphi(\tau, x ; X)$ is the corresponding loop phase. In terms of these two sided figures, see Fig. 7 , the loop additivity is realized by

$$
X_{1+2}\left(t_{1}+t_{2}, x\right)=X_{1}\left(t_{1}, x_{1}\right)+X_{2}\left(t_{2}, x_{2}\right)+C\left(l_{0}, l_{1}\right)+C\left(l_{1}, l_{2}\right)+C\left(l_{2}, l_{0}\right) .
$$

The implied phase additivity now reads

$$
\varphi\left(t_{1}+t_{2}, x ; X_{1+2}\right)=\varphi\left(t_{1}, x_{1} ; X_{1}\right)+\varphi\left(t_{2}, x_{2} ; X_{2}\right)+P_{3}\left(x, x_{1}, x_{2}\right) .
$$

Equation (4.3) is the version of additivity found by Marinov. It is the use of the two sided loops $X$, rather than the three sided loops $\widetilde{L}$, that accounts for the extra triangular area $P_{3}$.

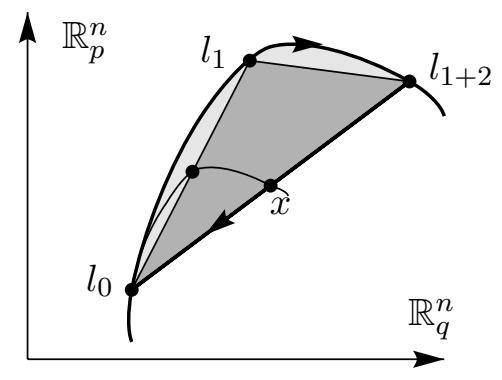

Figure 7. Marinov loop addition.

A somewhat similar additivity links the Schrödinger and Heisenberg phases with each other. On the left branch of Fig. $2, \varphi\left(t, x_{l} ; X_{l}\right)$ is defined by a two sided loop, $X_{l}\left(t, x_{l}\right)=T^{+}\left(l_{0}, l_{t}\right)+C\left(l_{t}, l_{0}\right)$. Similarly, $\varphi\left(t, x_{r} ; X_{r}\right)$ is the right branch area defined by $X_{r}\left(t, x_{r}\right)=T^{+}\left(r_{0}, r_{t}\right)+C\left(r_{t}, r_{0}\right)$. Recall from (A.5), that the four chord loop with midpoints $\left(x, x_{r}, x_{0}, x_{l}\right)$ has symplectic area $P_{4}\left(x, x_{r}, x_{0}, x_{l}\right)$. With this notation one has

Proposition 4. For $x \in \mathcal{N}_{t}$ let $\left(x, x_{r}^{j}, x_{0}^{j}, x_{l}^{j}\right)$ be the midpoints of the Heisenberg BC loop solution $\left\{W^{j}(t, x)\right\}_{1}^{N}$. Then

$$
\mathcal{A}\left(t, x ; W^{j}\right)=\varphi\left(t, x_{l}^{j} ; X_{l}^{j}\right)-\varphi\left(t, x_{r}^{j} ; X_{r}^{j}\right)+P_{4}\left(x, x_{r}^{j}, x_{0}^{j}, x_{l}^{j}\right), \quad j=1, \cdots, N .
$$

Proof. This follows directly from the loop decomposition

$$
\begin{aligned}
W(t, x) & =C\left(r_{0}, l_{0}\right)+T^{+}\left(l_{0}, l_{t}\right)+C\left(l_{t}, r_{t}\right)+T^{-}\left(r_{t}, r_{0}\right), \\
& =X\left(t_{1}, x_{l}\right)-X\left(t_{2}, x_{r}\right)+C\left(r_{0}, l_{0}\right)+C\left(l_{0}, l_{t}\right)+C\left(l_{t}, r_{t}\right)+C\left(r_{t}, l_{0}\right) .
\end{aligned}
$$




\subsection{Mutual Consistency of $U^{s c}(t)$ and $\rho^{s c}(t)$}

One may build the Heisenberg evolution $\rho(t, x)$ from its Schrödinger components, via $\rho(t, x)=U(t) * \rho_{0} * \overline{U(t)}$. The goal of this subsection is to show that the use of the approximation $U(t, x) \approx U^{s c}(t, x)$ combined with the evaluation of the $*$ product by the stationary phase approximation constructs $\rho^{s c}(t, x)$. This calculation will be carried out using the single sheet BC solutions found in Proposition 1. All the amplitudes and phases of $U^{s c}(t, x)$ and $\rho^{s c}(t, x)$ will be written without the multi-sheet subscript, $j$. This type of composition argument was used by Berry and Balazs [27] to construct $\rho^{s c}(t, x)$ in the case where $\mathbb{R}_{q}^{n}$ is one dimensional.

First observe that the required form of the $U^{s c}(t, x)$ is the Marinov version obeying the initial condition $U(t, x)=1$. Since $\Phi_{0}=0$, the amplitude takes the simplified form

$$
N(t, x)=\left[\operatorname{det} \frac{1}{2}\left(1+\nabla g\left(t \mid \widetilde{M}_{t}^{-1}(x)\right)\right)\right]^{-1 / 2} .
$$

Employing the three function $*$ product (A.8), the approximate $\rho(t, x)$ is

$$
\begin{aligned}
& {\left[U^{s c}(t) * \rho_{0} * \overline{U^{s c}(t)}\right](x) \equiv \mathcal{I}_{3}(t, x)} \\
& \quad=c \int U^{s c}\left(t, x_{1}\right) \rho\left(x_{2}\right) \overline{U^{s c}\left(t, x_{3}\right)} \delta\left(x_{1}-x_{2}+x_{3}-x\right) e^{i P_{3}\left(x_{1}, x_{2}, x_{3}\right) / \hbar} d x_{1} d x_{2} d x_{3}, \\
& \quad=c \int N\left(t, x_{1}\right) N\left(t, x_{3}\right) \alpha_{0}\left(x_{2}\right) e^{i\left[\varphi\left(t, x_{1}\right)-\varphi\left(t, x_{3}\right)+S_{0}\left(x_{2}\right)+P_{3}\left(x_{1}, x_{2}, x_{3}\right)\right] / \hbar} d x_{1} d x_{3} .
\end{aligned}
$$

where, $x_{2}=x_{1}+x_{3}-x$ and $c=(\pi \hbar)^{-2 n}$.

Denote the total phase in (4.6) by

$$
\Theta\left(x_{1}, x_{3} ; t, x\right)=\varphi\left(t, x_{1}\right)-\varphi\left(t, x_{3}\right)+S_{0}\left(x_{1}+x_{3}-x\right)+P_{3}\left(x_{3}, x, x_{1}\right) .
$$

Let $\xi=\left(x_{1}, x_{3}\right)$ and $\Theta^{\prime \prime}(\xi ; t, x)$ be the Hessian of $\Theta$ with respect to the $\xi$ variables. If $x_{1}=x_{1}(t, x)$ and $x_{3}=x_{3}(t, x)$ is an isolated non-degenerate critical point of $\Theta$, i.e. the solution of $\nabla_{\xi} \Theta(\xi ; t, x)=0$, then the standard [7] stationary phase approximation to (4.6) states

$$
\begin{aligned}
\mathcal{I}_{3}^{s t p h}(t, x)= & 2^{2 n} N\left(t, x_{1}\right) N\left(t, x_{3}\right) \alpha_{0}\left(x_{2}\right)\left|\operatorname{det} \Theta^{\prime \prime}\left(x_{1}, x_{3} ; t, x\right)\right|^{-1 / 2} \\
& \times \exp \left[\frac{i}{\hbar} \Theta\left(x_{1}, x_{3} ; t, x\right)+\frac{i \pi}{4} \operatorname{sgn} \Theta^{\prime \prime}\left(x_{1}, x_{3} ; t, x\right)\right]
\end{aligned}
$$

Above, the functional form of the constraint condition is $x_{2}(t, x)=x_{1}(t, x)+x_{3}(t, x)-x$.

In component form, the critical point equation reads

$$
\begin{aligned}
& x_{1}=x+\frac{1}{2} J \nabla \varphi\left(t, x_{3}\right)-\frac{1}{2} J \nabla S_{0}\left(x_{1}+x_{3}-x\right), \\
& x_{3}=x+\frac{1}{2} J \nabla \varphi\left(t, x_{1}\right)+\frac{1}{2} J \nabla S_{0}\left(x_{1}+x_{3}-x\right) .
\end{aligned}
$$

For small times and under the same assumptions as Proposition 1, one can show that the system above defines a contraction mapping and so has unique solution $x_{1}=x_{1}(t, x)$, $x_{3}=x_{3}(t, x)$. In fact the set of points $\left\{x_{2}(t, x), x_{1}(t, x), x, x_{3}(t, x)\right\}$ are exactly the four midpoints $\left\{x_{0}, x_{l}, x, x_{r}\right\}$ appearing in Fig. 2. The constraint condition for $x_{2}$ permits one 
to replace the $P_{3}\left(x_{3}, x, x_{1}\right)$ area by the $P_{4}\left(x_{3}, x, x_{1}, x_{2}\right)$, cf. (A.9). Substituting these critical point arguments into the total phase and using Proposition 4 one has

$$
\Theta\left(x_{1}(t, x), x_{3}(t, x) ; t, x\right)=S(t, x) .
$$

This demonstrates that the stationary phase produced by $U^{s c}(t) * \rho_{0} * \overline{U^{s c}(t)}$ is the same as the Heisenberg WKB phase $S(t, x)$.

Verifying amplitude consistency is more elaborate. A non-trivial determinant identity for $\Theta^{\prime \prime}$ in terms of the individual left-right flows is needed. Let $K_{l}=\nabla g(t \mid l), l=x_{2}(t, x)-$ $\frac{1}{2} \nabla J S_{0}\left(x_{2}(t, x)\right)$ and $K_{r}=\nabla g(t \mid r), r=x_{2}(t, x)+\frac{1}{2} \nabla J S_{0}\left(x_{2}(t, x)\right)$. Using identities obtained from (4.9) and (4.10) one can establish

$$
\begin{aligned}
\operatorname{det} & \Theta^{\prime \prime}\left(x_{1}(t, x), x_{3}(t, x) ; t, x\right) \\
& =2^{6 n}\left(\operatorname{det}\left(1+K_{l}\right)\left(1+K_{r}\right)\right)^{-1} \operatorname{det}\left[K_{l}\left(1-\frac{1}{2} J S_{0}^{\prime \prime}\left(x_{2}\right)\right)+K_{r}\left(1+\frac{1}{2} S_{0}^{\prime \prime}\left(x_{2}\right)\right)\right] .
\end{aligned}
$$

Putting (4.11) into (4.8) recovers the Theorem 2 amplitude $\alpha(t, x)$. In the small time sector all the determinants above are positive and $\operatorname{sgn} \Theta^{\prime \prime}\left(x_{1}, x_{3} ; t, x\right)=0$. Altogether one has the identity

$$
\left[U^{s c}(t) * \rho_{0} * \overline{U^{s c}(t)}\right]^{s t p h}(t, x)=\rho^{s c}(t, x) .
$$

\subsection{Quadratic Exactness}

If the Hamiltonian is a quadratic function, then the semiclassical quantities $U^{s c}(t, x)$ and $\rho^{s c}(t, x)$ are exact solutions of (1.1) and (1.2). Thus it is of interest to obtain the explicit formulas for the phases and amplitudes which arise is this special case.

The general time dependent Weyl symbol Hamiltonian of quadratic form is

$$
H(t, x)=\frac{1}{2} x \cdot H^{\prime \prime}(t) x+H^{\prime}(t) \cdot x,
$$

where $H^{\prime \prime}(t)$ is a $2 n \times 2 n$ symmetric matrix and $H^{\prime}(t)$ is a $2 n$ component vector. Both $H^{\prime \prime}(t)$ and $H^{\prime}(t)$ are real and $t$-continuous. The quantum Hamiltonian, $\widehat{H}(t)$, results if $x$ is replaced by $\hat{x}$ in $H(t, x)$.

The classical trajectories $g(t, s \mid x)$ generated by a quadratic $H(t, x)$ are linear functions of $x$. Explicit representations of $g(t, s \mid x)$ are obtained as follows.

The dynamical equations for $g(t, s \mid x)$ and $\nabla g(t, s \mid x)$ are the linear ODE's

$$
\begin{aligned}
& \frac{d}{d t} g(t, s \mid x)=J H^{\prime \prime}(t) g(t, s \mid x)+J H^{\prime}(t), \\
& \frac{d}{d t} \nabla g(t, s \mid x)=J H^{\prime \prime}(t) \nabla g(t, s \mid x),
\end{aligned}
$$

with initial conditions $g(s, s \mid x)=x$ and $\nabla g(s, s \mid x)=I$. Both (4.13) and (4.14) are Jacobi field equations, but (4.14) is homogeneous, while (4.13) has an inhomogeneity, $J H^{\prime}(t)$. Let $K(t, s)$ be the $S p(2 n)$ solution of

$$
\frac{d}{d t} K(t, s)=J H^{\prime \prime}(t) K(t, s), \quad K(s, s)=I .
$$


The solution of the system (4.15) is unique, which implies that $\nabla g(t, s \mid x)=K(t, s)$. In terms of $K(t, s)$, the forward $(t>s)$ and backward $(s<t)$ flows are

$$
\begin{aligned}
g(t, s \mid x) & =K(t, s)[x+F(t, s)], \\
g(s, t \mid x) & =K(s, t) x-F(t, s), \\
F(t, s) & =\int_{s}^{t} d \tau J K(\tau, s)^{T} H^{\prime}(\tau) \quad t \geq s .
\end{aligned}
$$

The solution (4.16) is verified by putting it into (4.13) and using the symplectic identity $K(t, s) J K(t, s)^{T}=J$. From (4.16) one obtains (4.17) by using the composition laws $g(t, s) \circ g(s, t)=I d$ and $K(s, t) K(t, s)=I$.

The behavior of the Heisenberg evolution is summarized in the following lemma. Note that for time dependent Hamiltonians the boundary condition problem needs to be stated in terms of $g(t, s)$. So the left side of (3.14) has two times, e.g. $M_{t, s}\left(x^{\prime}\right)$.

Lemma 5. For quadratic Hamiltonians the semiclassical density matrix is the exact solution of (1.2), $\rho^{s c}(t, x)=\rho(t, x)$. The structure of $\rho^{s c}(t, x)$ is given by:

(i) The $B C$ problem $M_{t, s}\left(x_{0}(t, x)\right)=x$ has a unique global solution given by the backward flow

$$
x_{0}(t, x)=K(s, t) x-F(t, s)
$$

$$
S(t, x)=S_{0}\left(x_{0}(t, x)\right), \quad \operatorname{det} \nabla M_{t, s}\left(x^{\prime}\right)=1
$$

$$
\rho^{s c}(t, x)=\rho_{0}\left(x_{0}(t, x)\right) e^{i S_{0}\left(x_{0}(t, x)\right) / \hbar} .
$$

Proof . The $x$-linearity of (4.16) combined with (3.14) implies $M_{t, s}\left(x^{\prime}\right)=g\left(t, s \mid x^{\prime}\right)$. Since $g(t, s)$ has inverse $g(s, t)$, (4.19) holds. The phase identity in (4.20) follows from the fact that chords evolve into chords, specifically $g\left(t, s \mid C\left(l_{0}, r_{0}\right)\right)=C\left(l_{t}, r_{t}\right)$. For this reason the Heisenberg loop $L(t, x)$ collapses to zero, so that all the WKB phase resides in the $S_{0}$ term. The determinant identity is a consequence of $\nabla M_{t, s}\left(x^{\prime}\right)$ being a symplectic matrix. That $\rho^{s c}(t, x)=\rho(t, x)$ is verified by direct substitution into (1.2).

The $x, y$ representation of the manifold $\Lambda_{t}$ is

$$
y(t, x)=\nabla S_{0}(K(s, t) x-F(t, s)) K(s, t) .
$$

This formula shows that $\Lambda_{t}$ is single sheeted. Statement (ii) establishes that $\Lambda_{t}$ is nonsingular for all $t$.

Next consider Schrödinger evolution. Let $\sigma(s) \equiv\{t \in \mathbb{R} \mid \operatorname{det}(K(t, s)-I)=0\}$ denote the set of caustic times where the amplitude function of $U^{s c}(t, x)$ diverges. The behavior of Schrödinger evolution is summarized below. These are known results ([10, 28, 29]) and their proof parallels that of Lemma 5 . 
Lemma 6. For quadratic Hamiltonian Schrödinger systems with initial data $U(0, x)=1$, the semiclassical solution of (1.1) is exact at all non-caustic times. The structure of $U^{s c}(t, x)=U(t, x)$ for $t \in \mathbb{R} / \sigma(s)$ is given by

(i) The $B C$ problem $\widetilde{M}_{t, s}\left(x_{0}(t, x)\right)=x$ has a solution given by:

$$
x_{0}(t, x)=(K(t, s)+I)^{-1}(2 x-F(t, s)),
$$

(ii)

(iii)

$$
N\left(t, x_{0}\right)=2^{n}|\operatorname{det}[K(t, s)+I]|^{-1 / 2}
$$

$$
\Phi(t, x)=-x \cdot J \frac{K(t, s)-I}{K(t, s)+I} x-2 J \frac{K(t, s)}{K(t, s)+I} F(t, s) x+C(t, s),
$$

where $C(t, s)$ is an $x$-independent constant.

The manifold $\Lambda_{t}$ is determined by the identity

$$
y(t, x)=-2 J \frac{1}{K(t, s)+I}[(K(t, s)-I) x+K(t, s) F(t, s)] .
$$

The $x$-linearity of $y(t, x)$ means that $\Lambda_{t}$ is a hyperplane. For each $t_{c} \in \sigma(s)$, where $K\left(t_{c}, s\right)$ has a -1 eigenvalue, $\Lambda_{t_{c}}$ is singular and $\left|y\left(t_{c}, x\right)\right|=\infty$. As $t \rightarrow t_{c}, U^{s c}(t, x)$ has a delta function behavior.

In comparing these Heisenberg and Schrödinger solutions, it is evident that the main difference is that $\rho(t, x)$ is always caustic free, while $U(t, x)$ has singular behavior at $t=t_{c}$.

\subsection{Evolution of semiclassically admissible observables}

An important special case of the Heisenberg evolution occurs when the initial operator $\hat{\rho}_{0}$ is semi-classically admissible, and as a result $S_{0}=0$. Most observables of physical interest (energy, momentum, angular momentum and their products) are semi-classically admissible.

How does Theorem 2 simplify in this case? First note that $S_{0}=0$ implies that the initial Lagrangian manifold $\Lambda_{0}=\mathbb{R}^{2 n}$. Furthermore, the BC equation (3.1) becomes $M_{t}\left(x^{\prime}\right)=g(t \mid x)=x$, and so $M_{t}^{-1}(x)=g(-t \mid x)$ for all $t, x$. From this it follows that $\operatorname{det} \nabla M_{t}\left(x^{\prime}\right)=1$ and so there are no caustics. Since $S_{0}=0$, the left, right and midpoint BC solutions are the same $x_{0}(t, x)=l_{0}(t, x)=r_{0}(t, x)$. This means the left, right, and midpoint flows collapse to a common trajectory and as a result the Heisenberg loop $L(t, x)$ reduces to a point. As a consequence $S(t, x)=0$. Altogether one has

$$
\rho^{s c}(t, x)=\rho_{0}(g(-t \mid x)) .
$$

This simple transport expression for $\rho^{s c}(t, x)$ is the point of departure [12, 13, 14, 15, 16] for higher order $\hbar$ expansions of $\rho(t, x)$. Identity (4.25) is an example of Egorov's theorem 30 .

The fact that $S(t, x)$ remains zero is in marked contrast to the behavior of $U^{s c}(t, x)$ where the phase $\Phi(t, x)$ spontaneously becomes non-zero even if its $t=0$ value vanishes. This Heisenberg null phase stability is a consequence of the symmetry $\mathcal{H}_{2}(x, y)=$ $-\mathcal{H}_{2}(x,-y)$. In turn, this symmetry means that $y(t)=y_{0}$ is a constant of motion for $\chi_{2}$ trajectories that have initial value $\left(x_{0}, y_{0}\right)=\left(x_{0}, 0\right)$. Equivalently, $\Lambda_{t}=\Lambda_{0}$. 


\section{Conclusions}

The representations of operators by Weyl symbols gives one a complete statement of quantum mechanics set in phase space. The Wigner transform is a Lie algebra isomorphism between operators (with commutator bracket) and symbols (with Moyal bracket). In this formalism, the Schrödinger and Heisenberg evolutions, $U(t, x)$ and $\rho(t, x)$, are generated by Hamiltonians that are pseudodifferential operators. These two problems are made amenable to semiclassical approximation by embedding them in an extended phase space $\chi_{2}=T^{*} \chi_{1}$. Set in $\chi_{2}$, the two evolution problems become special cases of an extended Schrödinger evolution.

The geometry of the WKB approximation is controlled by $\Lambda_{t}$, the dynamical Lagrangian manifold in $\chi_{2}$. The associated $\mathrm{BC}$ problem is solved in terms of loops built from chords and trajectories in PPS. The symplectic area of these loops form the WKB phase functions. The amplitudes are $\chi_{2}$ Poisson brackets constructed from the family of constraints that define the initial and final BC Lagrangian manifolds, $\Lambda_{0}$ and $\mathcal{N}_{t} \subseteq \mathbb{R}_{x}^{2 n}$.

An alternate characterization of Heisenberg evolution in symbol space is found in Marinov's 31] path integral representation of $\rho(t, x)$. This path integral has the following structure. Write the mapping $\rho_{0}(x) \mapsto \rho(t, x)$ in terms of an integral transform

$$
\rho(t, x)=\int d x^{\prime} K\left(t ; x, x^{\prime}\right) \rho_{0}\left(x^{\prime}\right)
$$

where the kernel $K$ is $h^{-n} \operatorname{Tr}\left[U(t) \Delta\left(x^{\prime}\right) U(t)^{\dagger} \Delta(x)\right]$. Define an action functional by

$$
\mathcal{S}(t ; \tilde{x}, \tilde{y})=\int_{0}^{t} d \tau\left[\tilde{y}(\tau) \cdot \dot{\tilde{x}}(\tau)-\mathcal{H}_{2}(\tilde{y}(\tau), \tilde{x}(\tau))\right]
$$

The path functions $\tilde{x}(\tau)$ obey the boundary conditions, $\tilde{x}(0)=x^{\prime}$ and $\tilde{x}(t)=x$, while the functions $\tilde{y}(\tau)$ are unrestricted. Then $K$ is the path integral,

$$
K\left(t ; x, x^{\prime}\right)=h^{-n} \iint D \tilde{x} D \tilde{y} e^{i \mathcal{S}(t ; \tilde{x}, \tilde{y}) / \hbar}
$$

Here $D \tilde{x} D \tilde{y}$ are infinite products of the dimensionless measures $h^{-n} d x$ and $h^{-n} d y$.

A second perspective of (5.2) follows from the extended Schrödinger equation (2.1). In the SPS setting, $\rho(t, x)$ may be interpreted as a wave function generated by the $\mathcal{H}_{2}$ evolution in $L^{2}\left(R_{x}^{2 n}\right)$. The kernel $K$ is then the $\chi_{1}$ coordinate space Dirac matrix element $\left\langle x\left|\exp \left(-i \widehat{\mathcal{H}}_{2} t / \hbar\right)\right| x^{\prime}\right\rangle$ and representation (5.2) is the corresponding Feynman path integral. The parallels with the $\rho^{s c}(t, x)$ approximation are evident. The functional $\mathcal{S}(t ; \tilde{x}, \tilde{y})$ has the same form as the generic WKB phase (3.3), but with $\tilde{y}(\tau), \tilde{x}(\tau)$ functional arguments replacing the classical flow $G\left(t \mid x^{\prime}, y^{\prime}\right)$.

The symplectic area WKB structure found for the evolutions $U(t)$ and $\rho(t)$ seems to be universal. An alternate version of Theorem 1, suitable for quantum systems with external electromagnetic fields, has been recently published in [32]. That paper introduced a gauge invariant $*$ product of the Berezin type. The $\left(x_{1}, x_{2}, x_{3}\right)$ triangle phase of this magnetic $*$ product is a symplectic area with respect to the Faraday 2 -form. The short time electromagnetic generalization of our Theorem 1 is found in Theorem 6 of [32]. Using 
the SPS methods developed here combined with the with symbol calculus of the magnetic * product, the extension of Heisenberg WKB representation of Theorem 2 to a gauge invariant form is straightforward.

Another direction of generalization is to consider non-Weyl $\hat{q}, \hat{p}$ quantum orderings. The simplest of these alternate ordering schemes [8] is

$$
\hat{f}(\hat{q}, \hat{p})=f(\alpha \stackrel{1}{\hat{q}}+(1-\alpha) \stackrel{3}{\hat{q}}, \stackrel{2}{\hat{p}}) .
$$

where $\alpha \in[0,1]$. Normal, anti-normal, and Weyl ordering occur for parameter values $\alpha=$ $0,1, \frac{1}{2}$. For all orderings with $\alpha \in(0,1)$ there will be symplectic area WKB representation of Schrödinger and Heisenberg evolutions.

The extended phase space method developed here is expected to be a good platform for further study. Since the $\chi_{2}$ Schrödinger evolution (2.1) is unitary in $L^{2}\left(\mathbb{R}_{x}^{2 n}\right)$, norm error bounds for $\left\|\rho(t)-\rho^{s c}(t)\right\|_{2}$ may be obtained with standard [7, 25] techniques. Higher order $\hbar$ expansions, with $U^{s c}(t, x)$ and $\rho^{s c}(t, x)$ as first terms, are straightforward to derive using the known higher order transport equations. Similarly the available globalization procedures [7, 8] readily apply in the $\chi_{2}$ framework.

\section{A The Star Product}

Comprehensive overviews of the Weyl symbol calculus are found in the books [18, 33]. In this appendix we summarize just the features of pseudodifferential operators and the star product that our semiclassical analysis requires. It is useful to begin with Weyl's original idea of quantization [3]. Let $\tilde{f}$ be the $\hbar$-Fourier transform of $f: T^{*} \mathbb{R}^{n} \rightarrow \mathbb{C}$

$$
f(x)=\int_{\mathbb{R}^{2 n}} d y \tilde{f}(y) \exp \left(\frac{i}{\hbar} y \cdot x\right) \equiv\left(F_{\hbar, y \rightarrow x} \tilde{f}\right)(x),
$$

then the Weyl quantization of $f$ is

$$
\widehat{f}=\int_{\mathbb{R}^{2 n}} d y \tilde{f}(y) \exp \left(\frac{i}{\hbar} y \cdot \hat{x}\right) \equiv f(\hat{q}, \hat{p})
$$

In this approach the family of unitary operators $\exp \frac{i}{\hbar} y \cdot \hat{x}$ is associated with the exponential functions $\exp \frac{i}{\hbar} y \cdot x$. By Fourier superposition this association is extended to construct a general operator $\widehat{f}$. The notation $\widehat{f}=f(\hat{q}, \hat{p})$ specifies a Weyl ordered function of the canonical operators $(\hat{q}, \hat{p})$.

Upon first inspection the quantization procedure above does not clarify if there is a well defined inverse: given an arbitrary operator $\widehat{f}$ on $\mathcal{H}$ how is $f(x)$ determined? Moyal solved this problem by showing that the inverse map $\widehat{f} \mapsto f$ is given by the Wigner transform

$$
f(x)=[\widehat{f}]_{\mathrm{w}}(x) \equiv \int d v e^{-p \cdot v / \hbar}\left\langle q+\frac{1}{2} v|\widehat{f}| q-\frac{1}{2} v\right\rangle
$$

Although there are a number of other consistent schemes [14 which associate functions on phase space with operators, normal ordering $[\hat{p}$ acts before $\hat{q}]$, Wick ordering $[(\hat{q}+i \hat{p})$ 
acts before $(\hat{q}-i \hat{p})]$, etc., Weyl quantization has the advantage that 1$)$ it treats $\hat{q}$ and $\hat{p}$ symmetrically, 2) self-adjoint operators have real symbols, and 3) the Moyal bracket is an even function of $\hbar$, in particular the semiclassical correction to the leading Poisson bracket term is $O\left(\hbar^{2}\right)$, not $O(\hbar)$.

A basic symmetry of the Wigner-Weyl isomorphism is its covariance with respect to affine canonical transformations. These transformations are parameterized by a matrix $R \in S p(2 n)$ and a displacement $x_{0}$,

$$
x^{\prime}=A(x)=R^{-1}\left(x-x_{0}\right), \quad A^{-1}\left(x^{\prime}\right)=R x^{\prime}+x_{0}^{\prime} .
$$

The pair $\left(R, x_{0}\right)$ also defines a family of unitary operators, $V=V\left(R, x_{0}\right)$ obeying

$$
V \hat{x} V^{-1}=A(\hat{x})=R^{-1}\left(\hat{x}-x_{0} I\right) .
$$

If $T\left(x_{0}\right)$ is the Heisenberg translation operator $\left(T\left(x_{0}\right)^{-1} \hat{x} T\left(x_{0}\right)=\hat{x}+x_{0} I\right)$ and $M(R)$ is a metaplectic operator (i.e. a unitary operator obeying $M^{-1} \hat{x} M=R \hat{x}$ ) then $V=$ $T\left(x_{0}\right) M(R)$. Affine covariance relates the Weyl symbol of a $V$ similarity transformed operator to its $A$ transformed symbol. Using the density matrix as an example, this universal covariance property [15] is

$$
\left(V \hat{\rho}(t) V^{-1}\right)_{\mathrm{w}} \equiv\left(\hat{\rho}(t)_{V}\right)_{\mathrm{w}}=\hat{\rho}(t)_{\mathrm{w}} \circ A^{-1} .
$$

In classical mechanics, an observable $f(x)$ is a real valued function and the product of observables is the commutative product of functions $f_{1}(x) f_{2}(x)$. In the Weyl symbol version of quantum mechanics, the commutative product becomes the non-commutative star product. There are three distinct types of representation of this star product; 1) the integral definition, 2) the left-right operator characterization, and 3) Groenewold's exponential derivative formula [15, 34]. Each of these three forms is useful in a different way. The integral form is convenient for proving the associativity of the $*$ product and determining the form of multiple products; the left-right forms represent the $*$ composition as a pseudo-differential operator (Lemma 8), and the Groenewold formula gives explicit coefficients [15] for the small $\hbar$-asymptotic expansion of the star product.

Consider first the integral representation. Let $\widehat{f}_{j}, j=1, \ldots, N$ be $N$ different operators. The Weyl symbol of their product is denoted by

$$
\left[\widehat{f}_{1} \widehat{f}_{2} \cdots \widehat{f}_{N}\right]_{\mathrm{w}}=f_{1} * f_{2} * \cdots * f_{N} .
$$

The higher order $*$ product requires the following two functions

$$
\begin{gathered}
P_{N}\left(x_{1}, x_{2}, \ldots, x_{N}\right)=2 \sum_{i=1}^{N-1} \sum_{j>i}^{N}(-1)^{i+j+1} x_{i} \wedge x_{j}, \quad x_{i} \wedge x_{j}=x_{i} \cdot J x_{j} \\
S_{N}\left(x_{1}, x_{2}, \ldots, x_{N}\right)=x_{1}-x_{2}+\cdots+(-1)^{N+1} x_{N} .
\end{gathered}
$$

Lemma 7. Let $f_{j} \in L^{1}\left(\mathbb{R}^{2 n}\right)$ be Weyl symbols, $j=1, \ldots, N$. For even $N \geq 2$, the integral form of the $*$ product is

$$
\left(f_{1} * f_{2} * \cdots * f_{N}\right)\left(x_{N+1}\right)=c^{N / 2} \int d x_{1} \ldots d x_{N} f_{1}\left(x_{1}\right) \cdots f_{N}\left(x_{N}\right) \exp \left[i P_{N+1}\left(x_{1}, \ldots, x_{N+1}\right) / \hbar\right],
$$


where $c=(\pi \hbar)^{-2 n}$. For odd $N \geq 3$,

$$
\begin{aligned}
\left(f_{1} * f_{2} * \cdots * f_{N}\right)\left(x_{N+1}\right)= & c^{(N-1) / 2} \int d x_{1} \ldots d x_{N} f_{1}\left(x_{1}\right) \cdots f_{n}\left(x_{N}\right) \\
& \delta\left(S_{N+1}\left(x_{1}, \ldots, x_{N+1}\right)\right) \exp \left[i P_{N}\left(x_{1}, \ldots, x_{N}\right) / \hbar\right] .
\end{aligned}
$$

Proof. The result for $N=2$ follows directly 15 from (A.1) and (A.2). The higher order products follow from the recurrence relation

$$
P_{N}\left(x_{1}, \ldots, x_{N}\right)=P_{N-1}\left(x_{1}, \ldots, x_{N-1}\right)+(-1)^{N+1} 2 x_{N} \wedge S_{N-1}\left(x_{1}, \ldots, x_{N-1}\right) .
$$

and an induction argument.

The phase $P_{N}$ in the $*$ product has a simple geometric meaning. It is the action line-integral

$$
P_{N}\left(x_{1}, \ldots, x_{N}\right)=\oint_{L_{N}} p \cdot d q=\int_{\Sigma_{N}} d q \wedge d p
$$

The geometric figure in the integral is an $N$-sided polygon in $T^{*} \mathbb{R}^{n}$ having $\left(x_{1}, \ldots, x_{N}\right)$ as the midpoints of its successive sides. Note that in Lemma the phase $P_{N}$ is needed only for $N$ odd. When $N$ is odd, this polygon is uniquely determined by the midpoints $\left(x_{1}, \ldots, x_{N}\right)$. Denote the boundary of this polygon by $L_{N}$ with orientation $x_{N} \rightarrow$ $x_{N-1} \cdots \rightarrow x_{1} \rightarrow x_{N} ; \Sigma_{N}$ is any oriented surface with outer boundary $L_{N}$. Thus $P_{N}$ is the symplectic area of this polygon. For a single star product $f_{1} * f_{2}$ the polygon is a triangle and $P_{3}$ is its symplectic area.

A variety of symmetries for the symplectic area, $P_{N}$, follow from its definition (A.5).

$$
\begin{aligned}
P_{N}\left(x_{1}+a, \ldots, x_{N}+a\right) & =P_{N}\left(x_{1}, \ldots, x_{N}\right)-\delta_{N} 2 a \wedge S_{N}\left(x_{1}, \ldots, x_{N}\right) \\
P_{N}\left(-x_{1},-x_{2}, \ldots,-x_{N}\right) & =P_{N}\left(x_{1}, x_{2}, \ldots, x_{N}\right) \\
P_{N}\left(x_{2}, x_{3}, \ldots, x_{N}, x_{1}\right) & =P_{N}\left(x_{1}, x_{2}, \ldots, x_{N}\right) \\
P_{N}\left(x_{N}, x_{N-1}, \ldots, x_{1}\right) & =-P_{N}\left(x_{1}, x_{2}, \ldots, x_{N}\right) .
\end{aligned}
$$

The above identities express translation, reflection, cyclic and anti-cyclic permutation invariance. The quantity $\delta_{N}$ is 1 if $N$ is even and zero otherwise.

These geometrical results for $N=3$ are due to Berezin [35]. It is now known [36] that the $*$ product on all symmetric symplectic spaces has a phase that is a triangle related symplectic area. Other representations of the $N$-order $*$ product are found in references [15, 23.

A second perspective on the $*$ product is to understand it as a $\Psi D O$. These operators are generated by smooth SPS functions, which are also called symbols. A $\Psi$ DO acting on the function $f(x)$ in denoted by $\mathcal{H}(\stackrel{2}{X}, \stackrel{1}{Y}) f(x)$. This operator is normal ordered, which means that $Y=-i \hbar \nabla_{x}$ acts first followed by $X$, which is multiplication by $x$. The operator $\mathcal{H}(\stackrel{2}{X}, \stackrel{1}{Y})$ is formally defined as follows,

$$
[\mathcal{H}(\stackrel{2}{X}, \stackrel{1}{Y}) f](x)=\left[F_{\hbar, y \rightarrow x} \mathcal{H}(x, y) F_{\hbar, x \rightarrow y} f\right](x)
$$


The presence of $\hbar$ in the Fourier transform $F_{\hbar, x \rightarrow y}$ means that $\mathcal{H}(\stackrel{2}{X}, \stackrel{1}{Y})$ is a function of $\hbar$, and that the $\hbar \rightarrow 0$ asymptotics is easy to study. Passing from a normal ordered symbol to a Weyl ordered symbol is easy and is achieved by the transformation

$$
\mathcal{H}_{W e y l}(x, y)=\exp \left(\frac{\hbar}{2 i} \nabla_{x} \cdot \nabla_{y}\right) \mathcal{H}(x, y)
$$

In this paper the symbols with SPS arguments $(x, y)$ generally correspond to normal ordered operators, while symbols with the PPS argument $x$ correspond to Weyl ordering.

The definition of $\mathcal{H}(\stackrel{2}{X}, \stackrel{1}{Y})$ is completed by placing $\mathcal{H}$ in a suitable class of functions. Two important function classes enter in Maslov's proof of (3.1). The first is $T^{m}\left(\mathbb{R}_{z}^{4 n}\right)$. This class denotes those $C^{\infty}\left(\mathbb{R}_{z}^{4 n}\right)$ functions $\mathcal{H}$ which satisfy the uniform growth estimates

$$
\left|\nabla_{x}^{\gamma} \nabla_{y}^{\rho} \mathcal{H}(x, y)\right| \leq C_{\gamma \rho}(1+|x|)^{m}(1+|y|)^{m}, \quad|\gamma|,|\rho| \geq 0, \quad(x, y) \in \mathbb{R}_{x}^{2 n} \times \mathbb{R}_{y}^{2 n}=\mathbb{R}_{z}^{4 n}
$$

where integer $m \geq 0$ and the positive constants $C_{\gamma \rho}$ are independent of $(x, y)$. The symbols in the class $T^{m}\left(\mathbb{R}_{z}^{4 n}\right)$ are $\hbar$ independent. Now widen this class so as to include semiclassically admissible operators. These operators depend on $\hbar$ and admit an asymptotic expansion about $\hbar=0$. One says $\mathcal{H}(x, y ; \hbar)$ belongs to the class $T_{+}^{m}\left(\mathbb{R}_{z}^{4 n}\right)$, if $\mathcal{H} \in C^{\infty}\left(\mathbb{R}_{z}^{4 n} \times \mathbb{R}^{+}\right)$and has the $\hbar$ expansion

$$
\mathcal{H}(x, y ; \hbar)=\sum_{j=0}^{J}(i \hbar)^{j} \mathcal{H}_{j}(x, y)+\hbar^{J+1} \mathcal{R}_{J}(x, y ; \hbar)
$$

The coefficient functions $\mathcal{H}_{j} \in T^{m}$, and the remainder term $\mathcal{R}_{J}$ has the $(x, y ; \hbar)$ uniform bound

$$
\left|\nabla_{x}^{\gamma} \nabla_{y}^{\rho}\left(\frac{\partial}{\partial \hbar}\right)^{\lambda} \mathcal{R}_{J}(x, y ; \hbar)\right| \leq C_{\gamma \rho \lambda}(1+|x|)^{m}(1+|y|)^{m}, \quad|\gamma|,|\rho|, \lambda \geq 0 .
$$

Now return to the $*$ product. Its left-right multiplication forms are the following.

Lemma 8. For all $f_{1}, f_{2} \in S^{\infty}\left(\mathbb{R}^{2 n}\right)$

$$
\begin{aligned}
& f_{1} * f_{2}(x)=f_{1}\left(\stackrel{2}{X}-\frac{1}{2} J \stackrel{1}{Y}\right) f_{2}(x), \\
& f_{1} * f_{2}(x)=f_{2}\left(\stackrel{2}{X}+\frac{1}{2} J \stackrel{1}{Y}\right) f_{1}(x) .
\end{aligned}
$$

Proof. Make a change of variables in (A.7) so that

$$
f_{1} * f_{2}(x)=(2 \pi \hbar)^{-2 n} \int d x_{2} d y_{2} f_{1}\left(x-\frac{1}{2} J y_{2}\right) f_{2}\left(x_{2}\right) \exp \left(\frac{i}{\hbar} y_{2} \cdot\left(x-x_{2}\right)\right)
$$

In the integral one can replace $f_{1}\left(x-1 / 2 J y_{2}\right)$ with $f_{1}(\stackrel{2}{X}-1 / 2 J \stackrel{1}{Y})$. Moving this last factor outside the integral gives (A.18). A similar argument gives (A.19).

Further review of the left right operator method in quantization and the use of the left right projections to provide representations of SPS is found in [18]. 


\section{B Jacobi Field Applications}

The Jacobi field of a classical system characterizes its stability. Given a trajectory $g(t \mid x)$, the $2 n \times 2 n$ matrix solution of the linear system

$$
\begin{gathered}
\frac{d}{d t} \nabla g(t \mid x)-J H^{\prime \prime}(g(t \mid x)) \nabla g(t \mid x)=0, \\
\nabla g(0 \mid x)=I,
\end{gathered}
$$

defines the associated Jacobi field. The solutions of (B.1, B.2) are symplectic matrices. For small times $\nabla g(t \mid x)$ will not differ greatly from its initial value $I$. This is made explicit in the following estimates. The norm $\|\cdot\|$ denotes the operator norm on $\mathbb{R}^{2 n}$.

Lemma 9. Let the Hessian of $H$ have the $x$-uniform bound $\left\|H^{\prime \prime}(x)\right\| \leq c_{1}<\infty$, then the Jacobi field has the growth estimates

$$
\|\nabla g(t \mid x)\| \leq e^{c_{1} t}, \quad\|\nabla g(t \mid x)-I\| \leq\left(e^{c_{1} t}-1\right) .
$$

Proof. Define $\xi(t \mid x)=\|\nabla g(t \mid x)\|$ and use $\frac{d}{d t}\|\nabla g(t \mid x)\| \leq\left\|\frac{d}{d t} \nabla g(t \mid x)\right\|$. Thus equation (B.1) and the Hessian bound implies

$$
\begin{gathered}
\frac{d}{d t} \xi(t \mid x) \leq\left\|J H^{\prime \prime}(g(t \mid x))\right\| \xi(t \mid x) \leq c_{1} \xi(t \mid x), \\
\xi(t \mid x) \leq e^{c_{1} t} .
\end{gathered}
$$

Now estimate $\|\nabla g(t \mid x)-I\|$. The integral equation equivalent of (B.1, B.2) is

$$
\nabla g(t \mid x)=I+\int_{0}^{t} J H^{\prime \prime}(g(\tau \mid x)) \nabla g(\tau \mid x) d \tau .
$$

Taking the norm of this relation gives

$$
\|\nabla g(t \mid x)-I\| \leq \int_{0}^{t}\left\|J H^{\prime \prime}(g(\tau \mid x))\right\| \xi(\tau \mid x) d \tau \leq c_{1} \int_{0}^{t} e^{c_{1} t} d \tau=\left(e^{c_{1} t}-1\right)
$$

Next we consider the detailed linkage between the BC problem, finite WKB amplitudes, and the non-singular set of $\Lambda_{t}$ with respect to the projection $\Pi_{1}$. Although this connection is implicit in the Maslov-Fedoriuk book [7], its precise statement is important for the BC solvability analysis and the interpretation of the WKB amplitude singularities.

First let us collect several definitions and facts. Recall that $D_{0}$ is the support of $S_{0}$ or $\Phi_{0}$. Furthermore the projection $\Pi_{1}: \Lambda_{0} \rightarrow D_{0}$ is diffeomorphic, with explicit inverse $\Pi_{1}^{-1}\left(x^{\prime}\right)=\left(x^{\prime}, \nabla S_{0}\left(x^{\prime}\right)\right), x^{\prime} \in D_{0}$. The regular subset of $\Lambda_{t}=G(t) \Lambda_{0}$ is $\mathcal{R}_{t}=\Lambda_{t} / \Sigma\left(\Lambda_{t}\right)$. Let $T_{m}\left(\Lambda_{t}\right)$ denote the tangent plane at $m \in \Lambda_{t}$. The statement, $m \in \mathcal{R}_{t}$, means that no vector in $T_{m}\left(\Lambda_{t}\right)$ has a zero $\Pi_{1}$ projection, i.e. $\operatorname{dim} \Pi_{1} T_{m}\left(\Lambda_{t}\right)=2 n$. Since $\Lambda_{t}$ is a Lagrangian manifold, $T_{m}\left(\Lambda_{t}\right)$ is a $2 n$ dimensional symplectic vector space for each $m$. With this notation we have 
Lemma 10. Let $x^{\prime} \in D_{0}=\Pi_{1} \Lambda_{0}$, then

$$
\operatorname{det} \nabla M_{t}\left(x^{\prime}\right) \neq 0 \quad \Leftrightarrow \quad G\left(t \mid x^{\prime}, \nabla S_{0}\left(x^{\prime}\right)\right) \in \mathcal{R}_{t} .
$$

Proof. The $\chi_{2}$ Jacobi field $\nabla G\left(t \mid m^{\prime}\right)$ maps vectors on $T_{m^{\prime}}\left(\Lambda_{0}\right)$ into vectors on $T_{G\left(t \mid m^{\prime}\right)}\left(\Lambda_{t}\right)$. The matrix $\nabla G\left(t \mid m^{\prime}\right)$ is symplectic, specifically $\operatorname{det} \nabla G\left(\tau \mid m^{\prime}\right)=1$. Let $\left\{e_{i}\right\}_{1}^{2 n}$ be a basis of $D_{0} \subseteq \mathbb{R}^{2 n}$ with origin at $x^{\prime}$. Then $\left\{W_{i}=\left(e_{i}, S_{0}^{\prime \prime}\left(x^{\prime}\right) e_{i}\right)\right\}_{1}^{2 n}$ is a basis of $T_{m^{\prime}}\left(\Lambda_{0}\right), m^{\prime}=$ $\left(x^{\prime}, \nabla S_{0}\left(x^{\prime}\right)\right)$. Since $\nabla G\left(t \mid m^{\prime}\right)$ is invertible $\left\{\nabla G\left(t \mid m^{\prime}\right) W_{i}\right\}_{1}^{2 n}$ is a basis of $T_{G\left(t \mid m^{\prime}\right)}\left(\Lambda_{t}\right)$. The Jacobi field for $\mathcal{H}_{2}$ flow is

$$
\nabla G\left(\tau \mid x^{\prime}, y^{\prime}\right)=\left[\begin{array}{cc}
\frac{1}{2}\left[\nabla g\left(t \mid l^{\prime}\right)+\nabla g\left(t \mid r^{\prime}\right)\right] & \frac{1}{4}\left[-\nabla g\left(t \mid l^{\prime}\right)+\nabla g\left(t \mid r^{\prime}\right)\right] J \\
J\left[\nabla g\left(t \mid l^{\prime}\right)-\nabla g\left(t \mid r^{\prime}\right)\right] & -\frac{1}{2} J\left[\nabla g\left(t \mid l^{\prime}\right)+\nabla g\left(t \mid r^{\prime}\right)\right] J
\end{array}\right] .
$$

Consider the conditions which ensure that $m \in \mathcal{R}_{t}$. Let $Z=\left(Z_{x}, Z_{y}\right)$ represent an arbitrary non-zero vector in $T_{m}\left(\Lambda_{t}\right)$. Since $\left\{\nabla G\left(\tau \mid m^{\prime}\right) W_{i}\right\}_{1}^{2 n}$ is a basis one has

$$
Z=\sum_{i=1}^{2 n} \lambda_{i} \nabla G\left(\tau \mid m^{\prime}\right) W_{i}=\sum_{i=1}^{2 n} \lambda_{i} \nabla G\left(\tau \mid m^{\prime}\right)\left(\begin{array}{c}
e_{i} \\
S_{0}^{\prime \prime}\left(x^{\prime}\right) e_{i}
\end{array}\right),
$$

where at least one of the constants $\lambda_{i}$ are non-zero. Project $Z$ onto $\mathbb{R}_{x}$, giving

$$
\begin{gathered}
\Pi_{1} Z=Z_{x}=\frac{1}{2} \frac{\partial}{\partial x^{\prime}}\left[g\left(t \mid x^{\prime}-\frac{1}{2} J \nabla S_{0}\left(x^{\prime}\right)\right)+g\left(t \mid x^{\prime}+\frac{1}{2} J \nabla S_{0}\left(x^{\prime}\right)\right)\right] V, \\
\Pi_{1} Z=\nabla M_{t}\left(x^{\prime}\right) V, \quad V=\sum_{i=1}^{2 n} \lambda_{i} e_{i} \neq 0 .
\end{gathered}
$$

Thus a vector in $T_{m}\left(\Lambda_{t}\right)$ can have zero $\Pi_{1}$ projection iff $\operatorname{det} \nabla M_{t}\left(x^{\prime}\right)=0$.

With the replacement of $S_{0}$ by $\Phi_{0}$ and $M_{t}$ by $\widetilde{M}_{t}$, the equivalence (B.4) holds for the Schrödinger problem.

\section{A Poincaré-Cartan Identity}

The following identity is required in the proof of Theorem 2 .

Lemma 11. Let $\gamma_{0}$ be an arbitrary smooth curve from $x_{1}$ to $x_{2}$. Let $\sigma\left(t \mid \gamma_{0}\right)$ be the surface defined by the images of $\gamma_{0}$ under the flow $g\left(\tau, t_{0}\right), t_{0} \leq \tau \leq t$. Then

$$
\int_{\sigma\left(t \mid \gamma_{0}\right)} d q \wedge d p=\oint_{\partial \sigma\left(t \mid \gamma_{0}\right)} p \cdot d q=\int_{t_{0}}^{t}\left[H\left(\tau, g\left(\tau, t_{0} \mid x_{1}\right)\right)-H\left(\tau, g\left(\tau, t_{0} \mid x_{2}\right)\right)\right] d \tau .
$$

Proof sketch. Decompose the curve $\gamma_{0}$ into segments that are singularity free with respect to some Lagrangian plane. For notational convenience denote this plane by $\mathbb{R}_{q}^{n}$. Now locally embed this curve into a non-singular Lagrangian manifold $\lambda_{0}$ having phase $s_{0}(q)$. Denote by $\lambda_{t}=g\left(t, t_{0} \mid \lambda_{0}\right)$ the time evolution of $\lambda_{0}$. Extend the original phase space 
$\chi_{1}$ by adding time to the $q$-coordinate manifold, $\left(q, t ; p, p_{t}\right) \in \chi_{1}^{+}=T^{*} R^{n+1}$. Let $s(t, q)$ be the phase of $\lambda_{t}$, then the manifold

$$
\lambda^{+} \equiv\left\{\left(q, t ; p, p_{t}\right) \in \chi_{1}^{+} \mid\left(p, p_{t}\right)=\left(\nabla s(t, q), \partial_{t} s(t, q)\right)\right\}
$$

is Lagrangian. On $\lambda^{+}$the 1 -form $\omega^{+}=p d q-H(t, q, p) d t$ is closed by virtue of the $\mathrm{H}-\mathrm{J}$ equation. Closed loop integrals on $\lambda^{+}$vanish and can be arranged to give (C.1).

Acknowledgments. The authors are indebted to M. V. Karasev for suggesting a number of improvements to the paper. We are also grateful to our colleagues Steve Fulling and Frank Molzahn for critically reading the manuscript. The research of T.A.O. is supported by a grant from Natural Sciences and Engineering Research Council of Canada.

\section{References}

[1] J. E. Moyal. Quantum mechanics as a statistical theory. Proc. Cambridge Philos. Soc., 45:99-124, 1949.

[2] E. P. Wigner. On the quantum correction for thermodynamic equilibrium. Phys. Rev., 40:749-759, 1932.

[3] H. Weyl. Quantum mechanics and group theory. Zeitschr. f. Phys., 46:1-46, 1927.

[4] A. Grossmann. Parity operator and quantization of $\delta$-functions. Commun. Math. Phys., 48:191-194, 1976.

[5] A. Royer. Wigner function as the expectation value of a parity operator. Phys. Rev. A, 15:449-450, 1977.

[6] A. Grossmann and P. Huguenin. Group-theoretical aspects of the Wigner-Weyl isomorphism. Helvetica Physica Acta, 51:252-261, 1978.

[7] V. P. Maslov and M. V. Fedoriuk. Semiclassical Approximation in Quantum Mechanics. Reidel, Dordrecht, 1981.

[8] M. V. Karasev and V. E. Nazaikinskii. On quantization of rapidly oscillating symbols. Math. USSR-Sb., 34:737-764, 1978.

[9] M. V. Berry. Semi-classical mechanics in phase space: a study of Wigner's function. Phil. Trans. R. Soc. Lond. A, 287:237-271, 1977.

[10] M. S. Marinov. An alternative to the Hamilton-Jacobi approach in classical mechanics. J. Phys. A: Math. Gen, 12:31-47, 1979.

[11] M. A. Antonets. The classical limit of solutions of the Cauchy problem for the Heisenberg-Weyl evolutional problem. Moscow Univ. Math. Bull, 2:21-27, 1977.

[12] R. T. Prosser. On the correspondence between classical and quantum mechanics. J. Math. Phys., 24:548-552, 1983. 
[13] D. Robert. Autour de l'Approximation Semi-Classique. Birkhäuser, Boston, 1978.

[14] F. A. Berezin and M. A. Shubin. The Schrödinger Equation. Kluwer, Dortrecht, 1991.

[15] T. A. Osborn and F. H. Molzahn. Moyal quantum mechanics: The semiclassical Heisenberg dynamics. Ann. Phys. (N.Y.), 241:79-127, 1995.

[16] B. R. McQuarrie, T. A. Osborn, and G. C. Tabisz. Semiclassical Moyal quantum mechanics for atomic systems. Phys. Rev. A, 58:2944-2961, 1998.

[17] E. J. Heller. Wigner phase space method: Analysis for semiclassical applications. J. Chem. Phys., 65:1289-1298, 1976.

[18] M. V. Karasev and V. P. Maslov. Nonlinear Poisson Brackets. Geometry and Quantization, volume 119 of Ser. Translations of Mathematical Monographs. AMS, Providence, 1993.

[19] R. Littlejohn. Semiclassical structure of trace formulas. J. Math. Phys., 31:29522977, 1990.

[20] J. P. Amiet and P. Huguenin. Generating functions of canonical maps. Helvetica Physica Acta, 53:377-397, 1980.

[21] M. V. Berry. Quantum scars of classical closed orbits in phase space. Proc. R. Soc. Lond. A, 42:219-231, 1989.

[22] A. M. Ozorio de Almeida and J. H. Hannay. Geometry of two dimensional tori in phase space: projections, sections and the Wigner function. Ann. of Phys., 139:115$154,1982$.

[23] A. M. Ozorio de Almeida. The Weyl representation in classical and quantum mechanics. Phys. Rep., 295:265-342, 1998.

[24] A. Weinstein. Symplectic manifolds and their Lagrangian submanifolds. Advances in Math., 6:329-346, 1971.

[25] A. Intissar. A remark on the convergence of the Feynman path integral for the Weyl pseudo-differential operators on $R^{n}$. Comm. Part. Diff. Equat., 7:1403-1437, 1982.

[26] H. Poincaré. Mécanique Céleste, volume 3. Gauthier-Villars, Paris, 1899.

[27] M. V. Berry and N. L. Balazs. Evolution of semiclassical quantum states in phase space. J. Phys. A, 79:625-642, 1979.

[28] J. P. Amiet and P. Huguenin. Exact Wigner function of bicanonical unitary transformations. Helvetica Physica Acta, 57:35-62, 1984.

[29] M. Gadella, J. M. Gracia-Bondía, L. M. Nieto and J. C. Várilly. Quadratic Hamiltonians in phase-quantum mechanics. J. Phys. A: Math. Gen., 22:2709-2738, 1989. 
[30] Ju. V. Egorov. Canonical transformations and pseudodifferential operators. Trans. Moscow Math. Soc., 24:1-28, 1971.

[31] M. S. Marinov. A new type of phase-space path integral. Phys. Lett. A, 153:5-11, 1991.

[32] M. V. Karasev and T. A. Osborn. Symplectic area, quantization, and dynamics in electromagnetic fields. J. Math. Phys., 2002.

[33] G. B. Folland. Harmonic analysis in Phase Space. Princeton University Press, Princeton, 1989.

[34] H. J. Groenewold. On the principles of elementary quantum mechanics. Physica, 12:405-460, 1946.

[35] F. A. Berezin. Quantization. Math. USSR-Izv., 8:1109-1165, 1974.

[36] A. Weinstein. Traces and triangles in symmetric symplectic spaces. Contemp. Math., 179:262-27, 1994. 